\title{
Closed Unstretchable Knotless Ribbons and the Wunderlich Functional
}

\author{
Brian Seguin ${ }^{1} \cdot$ Yi-chao Chen $^{2} \cdot$ Eliot Fried $^{3}$
}

Received: 9 April 2020 / Accepted: 13 April 2020 / Published online: 22 May 2020

(C) The Author(s) 2020

\begin{abstract}
In 1962, Wunderlich published the article "On a developable Möbius band," in which he attempted to determine the equilibrium shape of a free standing Möbius band. In line with Sadowsky's pioneering works on Möbius bands of infinitesimal width, Wunderlich used an energy minimization principle, which asserts that the equilibrium shape of the Möbius band has the lowest bending energy among all possible shapes of the band. By using the developability of the band, Wunderlich reduced the bending energy from a surface integral to a line integral without assuming that the width of the band is small. Although Wunderlich did not completely succeed in determining the equilibrium shape of the Möbius band, his dimensionally reduced energy integral is arguably one of the most important developments in the field. In this work, we provide a rigorous justification of the validity of the Wunderlich integral and fully formulate the energy minimization problem associated with finding the equilibrium shapes of closed bands, including both orientable and nonorientable bands with arbitrary number of twists. This includes characterizing the function space of the energy functional, dealing with the isometry and local injectivity constraints, and deriving the Euler-Lagrange equations. Special attention is given to connecting edge conditions, regularity properties of the deformed bands, determination of the parameter space needed to ensure that the deformation is surjective, reduction in isometry constraints, and deriving matching conditions and jump conditions associated with the Euler-Lagrange equations.
\end{abstract}

Keywords Isometric deformation · Ruled surface $\cdot$ Dimensional reduction · Constrained variational problem · Euler-Lagrange equations · Möbius band

Communicated by Paul Newton.

$\bowtie \quad$ Eliot Fried

eliot.fried@oist.jp

1 Loyola University Chicago, Chicago, Illinois, United States

2 University of Houston, Houston, Texas, United States

3 Okinawa Institute of Science and Technology Graduate University, Onna, Japan 
Mathematics Subject Classification 74G65 · 74G99 $\cdot$ 51P05 $\cdot 53$ B50

\section{Introduction}

For the past half-century, efforts to find the equilibrium shapes of Möbius bands and various other ribbon-like objects made from materials like paper, which can be modeled as unstretchable two-dimensional sheets, have relied mainly on numerical strategies for minimizing the Wunderlich (1962) functional, ${ }^{1}$ the derivation of which can be viewed as beginning with the bending energy

$$
2 \mu \int_{\mathcal{S}} H^{2} \mathrm{~d} a
$$

for a developable surface $\mathcal{S}$ with bending modulus $\mu>0$, mean curvature $H$, and element of area $\mathrm{d} a$. Granted that $\mathcal{S}$ is a half-twist Möbius band with midline $\mathcal{M}$ of length $\ell$ and uniform width $2 b$, Wunderlich (1962) noted that $\mathcal{S}$ must lie on the rectifying developable of $\mathcal{M}$ and on that basis concluded that the surface integral (1) reduces without approximation to a line integral

$$
\frac{\mu}{2} \int_{0}^{\ell} \bar{\kappa}_{0}^{2} V \log \frac{V+b}{V-b} \mathrm{~d} s,
$$

where $\bar{\kappa}_{0}$ is the restriction to $\mathcal{M}$ of the nonvanishing principal curvature of $\mathcal{S}, V$ is the point of regression of the generatrix of $\mathcal{S}$, and $\mathrm{d} s$ is the element of arclength along $\mathcal{M}$. In (2), it is tacit that the curvature of $\mathcal{S}$ is at least piecewise continuous. Wunderlich (1962) worked with dimensionless integrals that differ from (1) and (2) by a factor of $2 / \mu$. We nevertheless refer to (2) as the Wunderlich functional.

The purpose of Wunderlich's (1962) paper was to find the equilibrium shape of a developable Möbius band made from a rectangular strip of paper. Following Sadowsky (1930) he promoted the idea of finding the equilibrium shape by using an energy minimization principle ${ }^{2}$. His derivation of (2) provided an important step in that direction. However, as he concluded, finding the final equilibrium shape "would have required considerable effort, which is why it must be left undone for the time being." The following comments provide a comprehensive understanding of this task and the effort needed to accomplish it.

- For Wunderlich (1962), the equilibrium shape under discussion is a solution to a variational problem equivalent to

$$
2 \mu \int_{\mathcal{S}} H^{2} \mathrm{~d} a=\min .
$$

However, Wunderluch did not mention that a proper formulation of the problem (3) should begin with first specifying a reference configuration $\mathcal{D}$ of the strip of paper

\footnotetext{
1 See Todres (2015) for a translation of Wunderlich's (1962) paper into English.

2 See Hinz and Fried (2015) for a translation of Sadowsky's (1930) paper into English.
} 
and then finding the entire family $\mathcal{F}$ of kinematically admissible deformations $\chi$ of $\mathcal{D}$. Since the mean curvature $H$ depends on $\chi$ implicitly through the current configuration $\mathcal{S}=\chi(\mathcal{D})$, the bending energy $E$ is a functional of the deformation $\chi$ :

$$
E(\chi)=2 \mu \int_{\mathcal{S}} H^{2} \mathrm{~d} a .
$$

The variational problem (3) therefore amounts to finding a deformation $\chi_{e}$ that complies with the condition

$$
E\left(\chi_{e}\right) \leq E(\chi), \quad \chi \in \mathcal{F}
$$

Specifying the family $\mathcal{F}$ of kinematically admissible deformations $\chi$ in (4) is essential in formulating the variational problem.

- Kinematic admissibility here means, among other things, that the deformed surface must be isometric to the flat strip, a requirement that stems from the material being unstretchable. This means that for a deformation $\chi$ to be kinematically admissible, it must be an isometry, as defined, for instance, by do Carmo (1976, $\S 4-2)$. Moreover, to disallow self-penetration of the surface, all deformations must be injective.

- To ensure that the strip deforms to a closed ribbon, certain matching edge conditions must be imposed on the class of admissible deformations. As Wunderlich (1962) put it, a Möbius band is formed "by connecting the narrow edges of a long, rectangular strip of paper so that all diagonally opposed vertices meet." However, Wunderlich (1962) did not explicitly invoke any such conditions when deriving (2), and moreover, it has been shown by Chen et al. (2018) that for nonclosed ribbons, (2) does not accurately represent the bending energy (1).

- Wunderlich (1962) used a "partially numerical, partially graphical procedure" to find an "approximate portrayal of the equilibrium model" but did not derive the Euler-Lagrange equations of an appropriately constrained version of the functional (2) whose solution determines the exact equilibrium shape of the Möbius band.

The study of unstretchable material surfaces has been taken up by others in the aftermath of the pioneering work of Wunderlich (1962). Starostin and van der Heijden (2007, 2015) minimized the bending energy (1) over the class of Möbius bands of a fixed length and width that lie on a rectifying developable surface and dimensionally reduced the bending energy to obtain the Wunderlich energy. They then used a higher-order variational approach introduced by Gay-Balmaz et al. (2012) to obtain the corresponding Euler-Lagrange equations and specialized those equations to Möbius bands with certain symmetries. To construct a rectifying developable surface from an energy minimizing midline, they implicitly ruled out situations where the curvature of the midline vanishes over any open interval. However, Sadowsky's (1930) construction of a Möbius band shows that there exist bands for which that assumption is violated. Hence, there exist Möbius bands made from unstretchable strips that do not lie on rectifying developable surfaces. The problem of minimizing the bending energy (4) over the collection of rectifying developable Möbius bands is therefore not the same as 
that of minimizing over all Möbius bands of a given length and width. While it might well be that a Möbius band with minimum bending energy lies on a rectifying developable, it is illegitimate to assume so a priori. The approach of Starostin and van der Heijden also yields only the shapes of Möbius bands with various symmetries without determining the underlying isometric deformations from a reference configuration. Moreover, the matter of injectivity is not considered.

There are also a series of papers where Hornung (2011a,b,c) studied the structure of isometric deformations that are locally injective and are minimizers of the bending energy (4). In particular, these papers show that locally the surface corresponding to the deformation with minimum energy has a parameterization satisfying certain conditions which can be interpreted as Euler-Lagrange equations for the bending energy. Although these equations provide a basis for establishing regularity results for extrema, they do not constitute a boundary-value problem for determining the deformation from a reference configuration. The relevance of Hornung's (2011a; 2011b; 2011c) analysis to the equilibrium shapes of Möbius strips was discussed by Bartels and Hornung (2015). More broadly, the study of isometric immersions has been a subject of recent interest in the mathematics community, as exemplified by the works of Pakzad (2004), Müller and Pakzad (2005), and Kirchheim (2003).

Dias and Audoly (2015) studied the closely related problem of finding the equilibrium shape of a thin, unstretchable, elastic ribbon that is obtained by isometrically deforming a flat reference ribbon which need not be rectangular, but which must have a unique, well-defined midline. They utilized a double parameterization to describe the deformation: one parameterization for the reference configuration and another for the deformed configuration. The parameterization for the deformed configuration allows for a dimensional reduction in the bending energy, leading to an energy similar in spirit to Wunderlich's but for a broader class of ribbons. The parameterizations utilized in this work do not necessarily completely cover the reference and current configurations. This results in a dimensionally reduced energy that may underestimate or overestimate the true bending energy of the ribbon, as demonstrated by Chen et al. (2018).

The present work is aimed at filling the gaps left by Wunderlich (1962), gaps that, as itemized above, have not yet been treated in the ensuing literature. Our work contains many novel contributions, the most salient of which we now list:

1. We show that a kinematically admissible deformation $\chi$ can be represented by two vector-valued functions of arclength: a directrix $\boldsymbol{d}$ and a generatrix $\boldsymbol{g}$, which satisfy certain constraints. By this, we mean that each kinematically admissible deformation induces a directrix and generatrix that satisfy the constraints, and conversely, that given a directrix and generatrix satisfying these constraints, it is possible to construct a surface $\mathcal{S}$ and a kinematically admissible deformation from $\mathcal{D}$ to $\mathcal{S}$.

2. We formulate explicit matching conditions that ensure that the short edges of the strip are smoothly joined to form a closed ribbon and consistently apply those conditions when deriving the dimensionally reduced bending energy.

3. We show that the bending energy (1) can be dimensionally reduced to a functional of the directrix $\boldsymbol{d}$ and the generatrix $\boldsymbol{g}$ and we establish the equivalence of that functional and the Wunderlich functional (2). 
4. We introduce Lagrange and Karush-Kuhn-Tucker multipliers to enforce the constraints on the the directrix $\boldsymbol{d}$ and generatrix $\boldsymbol{g}$, and we formulate an associated variational problem involving an augmented energy functional.

5. We use elementary methods to derive the Euler-Lagrange equations associated with our augmented energy functional, resulting in a system of ordinary differential equations amenable to numerical analysis.

We begin by identifying the family $\mathcal{F}$ of kinematically admissible deformations $\chi$, each of which is a mapping from a reference configuration $\mathcal{D}$, which we take to be a flat rectangle of length $\ell$ and width $2 b$, to a spatial configuration that must satisfy certain matching edge conditions. Each deformation $\chi$ is required to be an isometry, meaning that it preserves the length of material curves. We refer to such deformations as isometric deformations. Each such $\chi$ can be represented by a pair of ruled parameterizations, one for the reference configuration and the other for the spatial configuration, namely

$$
\hat{\boldsymbol{x}}(\alpha, \beta)=\boldsymbol{c}(\alpha)+\beta \boldsymbol{f}(\alpha) \text { and } \hat{\boldsymbol{r}}(\alpha, \beta)=\boldsymbol{d}(\alpha)+\beta \boldsymbol{g}(\alpha),
$$

where $\alpha$ and $\beta$ are the parameters for the referential and spatial configurations, $\boldsymbol{c}$ and $\boldsymbol{d}$ are, respectively, referential and spatial directrices, and $\boldsymbol{f}$ and $\boldsymbol{g}$ are, respectively, referential and spatial generatrices. The stipulation that $\chi$ be an isometric deformation amounts to a collection of conditions involving the referential and spatial directrices and generatrices, along with the derivatives of those quantities with respect to arclength. We find, however, that those conditions can be recast entirely in terms of an equivalent collection of purely spatial conditions. These conditions are

$$
\left|d^{\prime}\right|=1, \quad|g|=1, \quad d^{\prime \prime} \cdot g=0, \quad \text { and } \quad\left(d^{\prime} \times g\right) \cdot g^{\prime}=0,
$$

where a prime denotes differentiation with respect to the parameter $\alpha$. Whereas (7) 1 and $(7)_{2}$ simply require that the spatial directrix be parametrized by arclength and that the spatial generatrix be of unit magnitude, $(7)_{3}$ requires that the normal vector of the spatial directrix be orthogonal to the spatial generatrix and $(7)_{4}$ requires that $\mathcal{S}$ be developable. Hence, contrary to some statements in the literature, dictating that $\mathcal{M}$ be an inextensible space curve is not sufficient to ensure that a minimizer of (2) corresponds to an isometric deformation.

Restricting attention to situations where the deformation $\chi$ is such that the rulings which form the ribbon in any particular configuration do not intersect, we show that this requirement implies that

$$
\sin ^{4} \theta-b^{2} \theta^{\prime 2} \geq 0
$$

where $\theta$ is the angle between $\boldsymbol{d}^{\prime}$ and $\boldsymbol{g}$. Moreover, we show that the condition (8) ensures that $\chi$ is locally injective on the interior of $\mathcal{D}$. While there might be reasons to relinquish (8) in favor of the stronger requirement that $\chi$ be globally injective on the interior of $\mathcal{D}$, this restriction is difficult to implement so we settle here with imposing local injectivity. 
Although Wunderlich (1962) considered only half-twist Möbius bands, the steps leading from (1) to (2) are independent of the number of twists that $\mathcal{S}$ possesses. Granted that (2) can be used to calculate the bending energy (1) of a half-twist Möbius band $\mathcal{S}$, it should apply just as well if $\mathcal{S}$ is instead a closed orientable ribbon, with or without twist, or a Möbius band with more than one half-twist. Our choice of reference configuration accommodates all such possibilities. We find, however, that the explicit form of the referential directrix depends on whether $\mathcal{S}$ is orientable or nonorientable. In either case, the referential directrix includes not only the midline of the rectangle $\mathcal{D}$ but also segments of its short edges. If $\mathcal{S}$ is orientable, then those segments must lie on opposite sides of $\mathcal{M}$. If $\mathcal{S}$ is nonorientable, then those segments must lie on the same side of $\mathcal{M}$. These additional segments are needed to ensure that $\mathcal{D}$ and $\mathcal{S}$ are completely covered by rulings, and thus, as is crucial, that the deformation $\chi$ preserves the identity of material points.

The distinction between orientable and nonorientable closed ribbons is imposed through two alternative conditions that the deformation $\chi$ must satisfy on the short edges of $\mathcal{D}$. For either of these alternatives, $\chi$ maps the short edges of $\mathcal{D}$ to a single curve on $\mathcal{S}$ and thus can at best be injective on the interior of $\mathcal{D}$. The curve in question may be a ruling of $\mathcal{S}$, but this is not generally so. If that curve is a ruling of $\mathcal{S}$, then the reduction from (1) to (2) is trivial. We therefore focus on situations in which the short edges of $\mathcal{D}$ are not associated with a ruling.

Our approach affords a unified derivation of a dimensionally reduced version of the bending energy (1) regardless of the orientability of the closed ribbon $\mathcal{S}$. The resulting functional involves integration over the midline $\mathcal{M}$ of $\mathcal{S}$ and depends on both the parametrizations $\boldsymbol{d}$ and $\boldsymbol{g}$ of the spatial directrix and spatial generatrix. In contrast to being independent, the variations of $\boldsymbol{d}$ and $\boldsymbol{g}$ must be consistent with the spatial constraints (7) to ensure that $\chi$ is an isometric deformation. Using the relations

$$
\boldsymbol{d}^{\prime} \cdot \boldsymbol{g}=\cos \theta \quad \text { and } \quad V=\frac{\sin ^{2} \theta}{\theta^{\prime}}
$$

we prove with a change of variables that our dimensional reduction is equivalent to the Wunderlich functional (2). We therefore provide an independent basis for that functional and, in so doing, confirm that it can, as suggested above, be used to calculate the bending energy (1) of a closed orientable ribbon, with or without twist, or a Möbius band with any number of half-twists. In that regard, the number of twists that can be sustained without violating the requirement that the curvature of $\mathcal{S}$ be piecewise continuous or necessitating points of self-contact is not unlimited but depends on the half-width to length aspect ratio $b / \ell$. To deduce the dimensionally reduced bending energy hinges on appropriate extensions of the parametrization of $\mathcal{S}$, the precise features of which depend on the orientability of $\mathcal{S}$. In both cases, however, the extensions make use of the requirements involving rulings that terminate on the short ends of the reference region $\mathcal{D}$.

Having shown that (1) can be expressed as a functional of the functions $\boldsymbol{d}$ and $\boldsymbol{g}$ that parametrize the spatial directrix and spatial generatrix, we prove that knowledge of those quantities is sufficient to construct both a $C^{1}$ closed ribbon $\mathcal{S}$ with piecewise continuous curvature and an underlying isometric deformation $\chi$ from the rectangular 
strip $\mathcal{D}$ to $\mathcal{S}$. This construction relies on assuming that $\boldsymbol{d}$ and $\boldsymbol{g}$ satisfy the constraints (7) and the local injectivity condition (8).

To assure that constraints (7) and (8) are met, we introduce four Lagrange multipliers together with a Karush-Kuhn-Tucker multiplier and consider a correspondingly augmented version of our dimensionally reduced energy functional. We then derive the Euler-Lagrange equations arising from that functional. In addition to ordinary differential equations for $\boldsymbol{d}$ and $\boldsymbol{g}$, the Euler-Lagrange equations include matching conditions that apply at the point of the midline where the ends of $\mathcal{D}$ are joined and jump conditions that apply at points on $\mathcal{M}$ across which the curvature of $\mathcal{S}$ is discontinuous. The Euler-Lagrange equations must be augmented by the isometry constraints (7) and complementary slackness condition associated with the Karush-Kuhn-Tucker multiplier in conjunction with an additional condition which encodes the number of twists in $\mathcal{S}$ through a constraint on the self-linking number of its midline $\mathcal{M}$.

The remainder of the paper is organized as follows. In Sect. 2, we focus on kinematical issues. After introducing the notion of an isometric deformation $\chi$ from a flat rectangular strip $\mathcal{D}$ to a closed ribbon $\mathcal{S}$, we present the connecting edge conditions that decide whether or not $\mathcal{S}$ is orientable, explain the basis for treating $\mathcal{D}$ and $\mathcal{S}$ as ruled surfaces, consider the degree of regularity that is allowed if two rulings meet at a point on the boundary of $\mathcal{S}$, and define the explicit parametrizations of the referential directrix that we utilize in the orientable and nonorientable cases. Having presented ruled parametrizations for $\mathcal{D}$ and $\mathcal{S}$, we conclude this section by describing the extensions of the parametrization of $\mathcal{S}$ that are needed subsequently to achieve our dimensional reduction. In Sect. 3, we define the bending energy, conduct the dimensional reduction, and exhibit the calculations needed to convert our dimensional reduction to the Wunderlich functional. In Sect. 4, we consider questions related to the regularity of $\mathcal{S}$ and $\chi$, derive versions of the constraint of isometry in terms of the functions $\boldsymbol{d}$ and $\boldsymbol{g}$ that parametrize the spatial directrix and spatial generatrix, and show how $\mathcal{S}$ and $\chi$ can be constructed given knowledge of $\boldsymbol{d}$ and $\boldsymbol{g}$. In Sect. 5, we derive the Euler-Lagrange equations for the energy functional arising from augmenting our dimensionally reduced bending energy to account for the constraints that $\boldsymbol{d}$ and $g$ must satisfy to ensure that $\chi$ is locally injective and an isometry. We also provide interpretations of the matching conditions that apply at the point on the midline $\mathcal{M}$ of $\mathcal{S}$ where the end points of the midline of $\mathcal{D}$ are joined consistent with the connecting edge conditions. Finally, we summarize and discuss our most salient results in Sect. 6.

\section{Isometric Deformations}

Let $\mathcal{D}$ be a rectangular strip, boundary included, of length $\ell$ and width $2 b$ that is embedded in a three-dimensional Euclidean point space $\mathcal{E}$. Suppose that $\mathcal{D}$ is made from an unstretchable material, meaning that it can sustain only isometric deformations. Let $\chi$ be such a deformation, assumed to be $C^{2}$ on the interior of $\mathcal{D}$, and denote the surface in $\mathcal{E}$ corresponding to the spatial configuration of $\mathcal{D}$ by $\mathcal{S}=\chi(\mathcal{D})$. 


\subsection{Connecting Edge Conditions}

We consider only isometric deformations $\chi$ that are $C^{2}$ on the interior of $\mathcal{D}$ and satisfy certain connecting edge conditions. To describe those conditions, we choose an origin $\boldsymbol{o}$, a positively oriented orthonormal basis $\left\{\boldsymbol{t}_{1}, \boldsymbol{t}_{2}, \boldsymbol{t}_{3}\right\}$, and associated Cartesian coordinates $x_{i}=(\boldsymbol{x}-\boldsymbol{o}) \cdot \boldsymbol{\imath}_{i}, i=1,2,3$, whereby $\mathcal{D}$ can be identified with the twodimensional subset $[0, \ell] \times[0,2 b] \times\{0\}$ of $\mathcal{E}$. We then assume that $\mathcal{S}$ is a $C^{1}$ surface and that one of the connecting edge conditions

$$
\chi\left(0, x_{2}, 0\right)=\chi\left(\ell, x_{2}, 0\right), \quad x_{2} \in[0,2 b],
$$

or

$$
\chi\left(0, x_{2}, 0\right)=\chi\left(\ell, 2 b-x_{2}, 0\right), \quad x_{2} \in[0,2 b]
$$

holds. For either (10) or (11), the surface $\mathcal{S}$ is what we call a "closed ribbon." Whereas $\mathcal{S}$ is orientable for (10), it is nonorientable for (11). We allow for the possibility of twisting $\mathcal{D}$ any number of times in conjunction with the imposition of (10) or (11).

If $\mathcal{S}$ is orientable, then its boundary $\partial \mathcal{S}$ has two disjoint edges. If, otherwise, $\mathcal{S}$ is nonorientable, then $\partial \mathcal{S}$ has only a single edge. We restrict attention to situations where $\partial \mathcal{S}$ is free of both traction and couple traction regardless of the orientability of $\mathcal{S}$.

\subsection{Closed Ribbons as Ruled Surfaces}

Since the Gaussian curvature of $\mathcal{S}$ must vanish for any isometric deformation $\chi$ of $\mathcal{D}$, any closed ribbon $\mathcal{S}$ can be partitioned depending on whether or not its mean curvature $H$ vanishes. The work of Hartman and Nirenberg (1959) shows that the subset of $\mathcal{S}$ within which $H \neq 0$ can be covered by straight line segments, or rulings, which are asymptotic curves. Due to the rectangular shape of $\mathcal{D}$, the connected components of $\mathcal{S}$ where $H=0$ must be a trapezoid, a triangle, or an isolated line segment. With such a partition, $\mathcal{S}$ can be represented as a ruled surface. Specifically, any curved part of $\mathcal{S}$ is ruled by the corresponding asymptotic curves. Furthermore, any trapezoidal or triangular subset of $\mathcal{S}$ can be ruled in a way that ensures a continuous transition to the adjacent curved portions of $\mathcal{S}$ and an isolated line segment of $\mathcal{S}$ upon which $H=0$ automatically provides a smooth transition between the adjacent curved portions of $\mathcal{S}$. A complete ruling of $\mathcal{S}$ induces an associated ruling of $\mathcal{D}$ via the underlying isometric deformation $\chi$. Thus, since the connecting edge conditions (10) and (11) make it impossible for any particular ruling of $\mathcal{D}$ to span its entire length, each ruling of $\mathcal{S}$ must intersect the boundary $\partial \mathcal{S}$ of $\mathcal{S}$ at exactly two points.

Let the left- and right-hand edges, $\{0\} \times[0,2 b] \times\{0\}$ and $\{\ell\} \times[0,2 b] \times\{0\}$, of $\mathcal{D}$ be denoted by $\partial \mathcal{D}_{L}$ and $\partial \mathcal{D}_{R}$, respectively, and choose a fixed value of $x_{2}$ belonging to $[0,2 b]$. Then, if (10) holds, so that $\mathcal{S}$ is orientable, the slopes of the rulings that terminate at the points $x_{2} \boldsymbol{l}_{2}$ and $\ell \boldsymbol{t}_{1}+x_{2} \boldsymbol{l}_{2}$ on $\partial \mathcal{D}_{L}$ and $\partial \mathcal{D}_{R}$ must be identical, as depicted in Fig. 1a. Moreover, the deformed images of those rulings must meet on $\mathcal{S}$ to form a single ruling that contains the point $\chi\left(0, x_{2}, 0\right)=\chi\left(\ell, x_{2}, 0\right)$. Alternatively, 


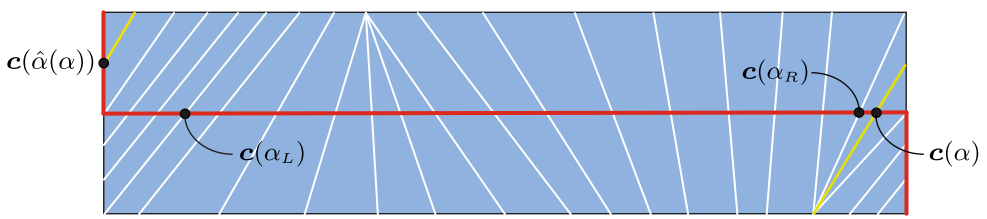

(a) Sample ruling of the rectangular strip $\mathcal{D}$ for the orientable connecting edge condition (2.1).

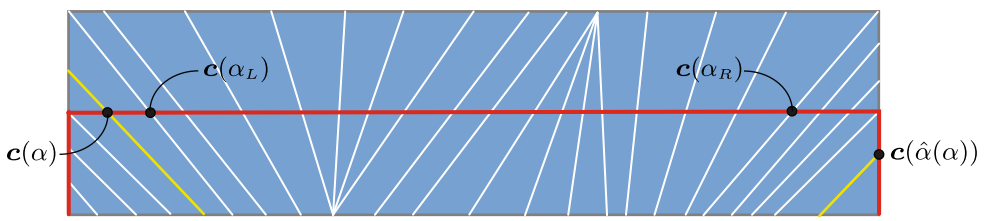

(b) Sample ruling of the rectangular strip $\mathcal{D}$ for the nonorientable connecting edge condition (2.2).

Fig. 1 Representative depictions of rulings of the rectangular strip $\mathcal{D}$ for the connecting edge conditions (10) and (11). The red curves indicate the directrices. Whereas the slopes of the yellow rulings containing the points $\boldsymbol{c}(\alpha)$ and $\boldsymbol{c}(\hat{\alpha}(\alpha))$ are equal for orientable bands, they are of equal magnitude but opposite sign for nonorientable bands. Hence, although the rulings passing through $\boldsymbol{c}(\alpha)$ and $\boldsymbol{c}(\hat{\alpha}(\alpha))$ are disjoint on $\mathcal{D}$, their images under the deformation $\chi$ join smoothly to form a single ruling on the band $\mathcal{S}=\chi(\mathcal{D})$. Restricting attention to deformations for which the rulings intersecting the right-hand edge of $\mathcal{D}$ have positive slopes

if (11) holds, so that $\mathcal{S}$ is nonorientable, the slopes of the ruling that terminate at the points $x_{2} \boldsymbol{l}_{2}$ and $\ell \boldsymbol{l}_{1}+\left(2 b-x_{2}\right) \boldsymbol{t}_{2}$ on $\partial \mathcal{D}_{L}$ and $\partial \mathcal{D}_{R}$ must differ only by their signs, as depicted in Fig. 1b. Moreover, the deformed images of those rulings must meet to form a single ruling on $\mathcal{S}$ that contains the point $\chi\left(0, x_{2}, 0\right)=\chi\left(\ell, 2 b-x_{2}, 0\right)$.

Let $\mathcal{J}$ denote the line segment on $\mathcal{S}$ along which $\partial \mathcal{D}_{L}$ and $\partial \mathcal{D}_{R}$ are joined via either of the connecting edge conditions (10) or (11). Then, $\mathcal{J}$ might or might not be a ruling of $\mathcal{S}$ and these alternatives are mutually exclusive. Since the situation in which $\mathcal{J}$ is a ruling of $\mathcal{S}$ is both atypical and straightforward to treat, we consider only the more prevalent and substantially more challenging alternative in which $\mathcal{J}$ is not a ruling of $\mathcal{S}$. If $\mathcal{J}$ is not a ruling of $\mathcal{S}$, then there is necessarily a family of rulings that cross $\mathcal{J}$. That being so, no generality is lost by considering deformations for which the rulings that intersect the right-hand edge of $\mathcal{D}$ have positive slopes as depicted in Fig. 1a, b.

\subsection{Kinematically Imposed Smoothness of the Deformation on the Boundary of the Strip}

Due to the restrictive nature of the class of isometric deformations, it can be shown that, in addition to being $C^{2}$ on the interior of the rectangular strip $\mathcal{D}$, the deformation $\chi$ from $\mathcal{D}$ to a band $\mathcal{S}$ is also $C^{2}$ at all points on the boundary $\partial \mathcal{D}$ of $\mathcal{D}$ at which rulings do not intersect. To verify this assertion, consider a point $\boldsymbol{x}_{\circ}$ on $\partial \mathcal{D}$ at which rulings do not intersect. Since the direction of the rulings is a continuous function of position, rulings that are sufficiently close to $\boldsymbol{x}_{\circ}$ can be extended slightly outside of $\mathcal{D}$ without intersecting. Thus, there is a small two-dimensional neighborhood $\mathcal{N}$ of $\boldsymbol{x}_{\circ}$, in the $x_{3}=0$ plane, that is covered by rulings that do not intersect. Since an isometric 
Fig. 2 Rulings in the triangular portion $\mathcal{T}_{r}$ of the strip $\mathcal{D}$ associated with a curved portion of $\mathcal{S}$ covered by rulings that intersect at a point on $\partial \mathcal{S}$
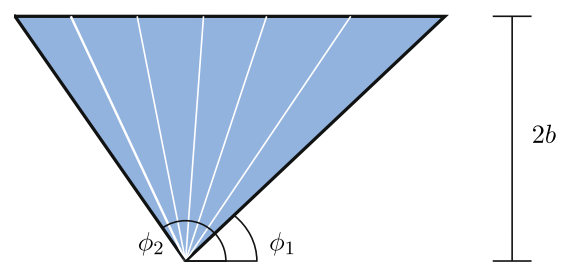

deformation $\chi$ transforms rulings rigidly, ${ }^{3} \chi$ has a natural isometric extension to $\mathcal{N}$. Moreover, by the construction of that extension, $\chi$ has the same regularity on $\mathcal{N}$ as it does on the interior of $\mathcal{D}$. Thus, the isometric extension of $\chi$ is $C^{2}$ on $\mathcal{N}$, and, consequently, the original deformation $\chi$ is itself $C^{2}$ at $\boldsymbol{x}_{\circ}$.

To illustrate that $\chi$ need not be smooth at any point of the boundary $\partial \mathcal{D}$ of the strip $\mathcal{D}$ at which rulings intersect, let $\mathcal{T}_{c}$ denote a curved portion of $\mathcal{S}$ covered by rulings that intersect at a point on $\partial \mathcal{S}$, and let $\mathcal{T}_{r}$ be the part of the rectangular strip $\mathcal{D}$ associated with $\mathcal{T}_{c}$. Notice that $\mathcal{T}_{r}$ is a triangular region, as depicted in Fig. 2. Thus, $\mathcal{T}_{r}$ can be parameterized by polar coordinates $(\phi, \rho)$, with the point where the rulings intersect serving as the origin, with $\phi$ denoting the angle measured counterclockwise from the $x_{1}$-axis, and $\rho$ measuring the radius from that origin. If $\boldsymbol{p}$ denotes the position on $\mathcal{S}$ where the rulings intersect, then the curved portion $\mathcal{T}_{c}$ of $\mathcal{S}$ under consideration can be parameterized by

$$
\tilde{\boldsymbol{r}}(\phi, \beta)=\boldsymbol{p}+\rho \boldsymbol{g}(\phi)
$$

where $g(\phi)$ is a unit vector in the direction of the ruling associated with the angle $\phi$. A unit normal $\boldsymbol{n}$ to $\mathcal{T}_{c}$ is then given by

$$
\boldsymbol{n}=\frac{\tilde{\boldsymbol{r}}_{\rho} \times \tilde{\boldsymbol{r}}_{\phi}}{\left|\tilde{\boldsymbol{r}}_{\rho} \times \tilde{\boldsymbol{r}}_{\phi}\right|} .
$$

As defined, $\boldsymbol{n}$ is independent of position along each ruling and, thus, is independent of $\beta$. Since $\mathcal{T}_{c}$ is a curved portion of $\mathcal{S}, \boldsymbol{n}$ cannot be uniform on $\mathcal{T}_{c}$. We thus see that $\mathcal{T}_{c}$ has rulings along which $\boldsymbol{n}$ takes different values. Let $\boldsymbol{n}_{1}$ and $\boldsymbol{n}_{2}$ denote the normal vectors on two such rulings. If $\chi$ were $C^{1}$, it would then follow that the unit normal to $\mathcal{S}$ would be continuous up to $\partial \mathcal{S}$, implying that $\boldsymbol{n}_{1}=\boldsymbol{n}_{2}$, which is incompatible with the curved nature of $\mathcal{T}_{c}$. We thus conclude, as claimed, that $\chi$ need not be $C^{1}$ at a point on $\partial \mathcal{D}$ where the rulings intersect.

\subsection{Parameterizations of the Strip and the Closed Ribbon}

In describing the rectangular strip $\mathcal{D}$ and the ribbon $\mathcal{S}$ as parametrized surfaces, it is necessary to consider whether the closed ribbon $\mathcal{S}$ is orientable or nonorientable. In either case, the parametrizations rest on a convenient identification of a curve $\mathcal{C}_{r}$ that

\footnotetext{
${ }^{3}$ See Chen et al. (2018, p. 154).
} 


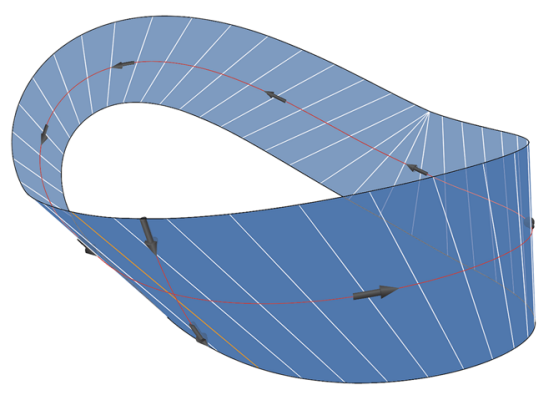

(a)

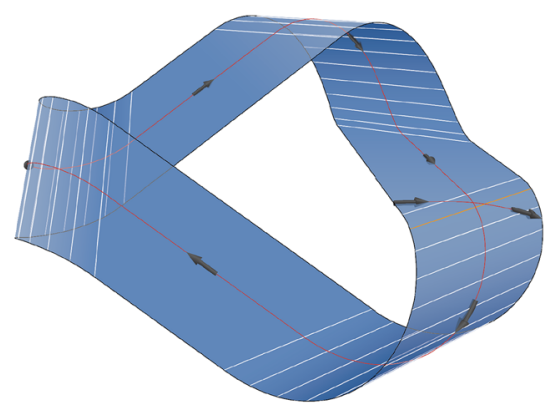

(b)

Fig. 3 Examples of (a) orientable and (b) nonorientable closed ribbons $\mathcal{S}$. The spatial directrix $\mathcal{C}_{S}$ of each ribbon $\mathcal{S}$ consists not only of the midline $\mathcal{M}$ of $\mathcal{S}$ but also of a geodesic that is the image of the subset $\{\boldsymbol{c}(\alpha) \mid \alpha \in[0, b) \cup(\ell+b, \ell+2 b]\}$ of the referential directrix $\mathcal{C}_{r}$ and connects two points on $\partial \mathcal{S}$

serves as a directrix for $\mathcal{D}$. If $\mathcal{S}$ is orientable, we take $\mathcal{C}_{r}$ to be parameterized by

$$
\boldsymbol{c}(\alpha)= \begin{cases}(2 b-\alpha) \boldsymbol{\iota}_{2}, & \alpha \in[0, b), \\ (\alpha-b) \boldsymbol{\iota}_{1}+b \boldsymbol{\iota}_{2}, & \alpha \in[b, \ell+b), \\ \ell \boldsymbol{\iota}_{1}+(\ell+2 b-\alpha) \boldsymbol{\iota}_{2}, & \alpha \in[\ell+b, \ell+2 b] .\end{cases}
$$

If, alternatively, $\mathcal{S}$ is nonorientable, we take $\mathcal{C}_{r}$ to be parameterized by

$$
\boldsymbol{c}(\alpha)= \begin{cases}\alpha \boldsymbol{\iota}_{2}, & \alpha \in[0, b), \\ (\alpha-b) \boldsymbol{\iota}_{1}+b \boldsymbol{\iota}_{2}, & \alpha \in[b, \ell+b), \\ \ell \boldsymbol{\imath}_{1}+(l+2 b-\alpha) \boldsymbol{\iota}_{2}, & \alpha \in[\ell+b, \ell+2 b]\end{cases}
$$

Notice that (14) and (15) are defined such that the referential directrix $\mathcal{C}_{r}$ is parametrized by arclength. In Fig. $1, \mathcal{C}_{r}$ is depicted in red. Regardless of the orientability of $\mathcal{S}$, each ruling of $\mathcal{D}$ intersects $\mathcal{C}_{r}$ exactly once.

The spatial directrix $\mathcal{C}_{s}$ is the image of $\mathcal{C}_{r}$ under the deformation $\chi$ from $\mathcal{D}$ to $\mathcal{S}$ and has arclength parameterization

$$
\boldsymbol{d}(\alpha)=\chi(\boldsymbol{c}(\alpha)), \quad \alpha \in[0, \ell+2 b],
$$

where $\boldsymbol{c}$ is determined by (14) or (15) depending on whether $\mathcal{S}$ is orientable or nonorientable. Notice that, as Fig. 3 shows, the midline $\mathcal{M}$ of $\mathcal{S}$ is parametrized by

$$
\boldsymbol{d}(\alpha)=\chi(c(\alpha)), \quad \alpha \in[b, \ell+b]
$$

and, thus, constitutes but a portion of $\mathcal{C}_{s}$.

For $\alpha \in[0, \ell+2 b]$, let $f(\alpha)$ denote the unit vector in the direction of the ruling that passes through $\boldsymbol{c}(\alpha)$ chosen so that $\left(\boldsymbol{c}^{\prime}(\alpha) \times \boldsymbol{f}(\alpha)\right) \cdot \boldsymbol{\iota}_{3}>0$, and let $\theta(\alpha)$ denote 
the angle between $\boldsymbol{c}^{\prime}(\alpha)$ and $\boldsymbol{f}(\alpha)$. Then, $\left\{\boldsymbol{c}^{\prime}, \boldsymbol{\iota}_{3} \times \boldsymbol{c}^{\prime}, \boldsymbol{\iota}_{3}\right\}$ is a Darboux frame for the referential directrix of $\mathcal{D}$ and it follows that $f$ has the representation

$$
\boldsymbol{f}=\cos \theta \boldsymbol{c}^{\prime}+\sin \theta \boldsymbol{\iota}_{3} \times \boldsymbol{c}^{\prime}
$$

where since the referential directrix consists of straight line segments and $\boldsymbol{t}_{3}$ is a fixed unit vector,

$$
c^{\prime \prime}=\mathbf{0} \quad \text { and } \quad\left(\iota_{3} \times c^{\prime}\right)^{\prime}=\boldsymbol{\imath}_{3}^{\prime} \times c^{\prime}+\boldsymbol{\iota}_{3} \times c^{\prime \prime}=\mathbf{0} .
$$

For each $\alpha \in[0, \ell+2 b]$, it can be shown that it is possible to find $\beta_{ \pm}(\alpha)$ such that $\boldsymbol{c}(\alpha)+\beta_{-}(\alpha) \boldsymbol{f}(\alpha)$ and $\boldsymbol{c}(\alpha)+\beta_{+}(\alpha) \boldsymbol{f}(\alpha)$ are the end points of the ruling that passes through the point $c(\alpha)$ on the referential directrix $\mathcal{C}_{r}$. It follows that there is a parameter set

$$
\mathcal{P}=\left\{(\alpha, \beta) \in \mathbb{R}^{2} \mid \alpha \in[0, \ell+2 b], \beta \in\left[\beta_{-}(\alpha), \beta_{+}(\alpha)\right]\right\},
$$

such that $\hat{\boldsymbol{x}}: \mathcal{P} \rightarrow \mathcal{D}$ defined by

$$
\hat{\boldsymbol{x}}(\alpha, \beta)=\boldsymbol{c}(\alpha)+\beta \boldsymbol{f}(\alpha), \quad(\alpha, \beta) \in \mathcal{P},
$$

covers $\mathcal{D}$. In a similar way, there is a function $\hat{\boldsymbol{r}}: \mathcal{P} \rightarrow \mathcal{S}$ of the form

$$
\hat{\boldsymbol{r}}(\alpha, \beta)=\boldsymbol{d}(\alpha)+\beta \boldsymbol{g}(\alpha), \quad(\alpha, \beta) \in \mathcal{P}
$$

which covers $\mathcal{S}$, where $\boldsymbol{g}(\alpha)=(\nabla \chi(\hat{\boldsymbol{x}}(\alpha, 0))) \boldsymbol{f}(\alpha)$ is a unit vector parallel to the ruling that passes through the point $\boldsymbol{d}(\alpha)$ on the spatial directrix $\mathcal{C}_{S}$.

Notice, from (21) and (22), that the parameterizations $\hat{\boldsymbol{x}}$ and $\hat{\boldsymbol{r}}$ determine the deformation $\chi$ through the requirement that

$$
\boldsymbol{r}=\boldsymbol{\chi}(\boldsymbol{x}) \quad \text { if and only if } \quad \boldsymbol{x}=\hat{\boldsymbol{x}}(\alpha, \beta) \text { and } \boldsymbol{r}=\hat{\boldsymbol{r}}(\alpha, \beta) .
$$

Due to the assumed smoothness of $\chi, \boldsymbol{d}$ is $C^{2}$ except at $\alpha=b$ and $\alpha=\ell+b$. Also, by a result due to Hartman and Wintner (1951), $\boldsymbol{f}$ and $\boldsymbol{g}$ are both $C^{1}$ except possibly at $\alpha=b$ and $\alpha=\ell+b$ and at values of $\alpha$ at which flat and curved portions of $\mathcal{S}$ meet. Chen et al. (2018) showed that the parameterizations (21) and (22) are subject to the following constraints, which are necessary and sufficient to guarantee that $\chi$ is an isometric deformation from $\mathcal{D}$ to $\mathcal{S}$ :

$$
\left.\begin{array}{c}
\left|\boldsymbol{c}^{\prime}\right|=\left|\boldsymbol{d}^{\prime}\right|=1, \quad|\boldsymbol{f}|=|\boldsymbol{g}|=1, \quad\left|\boldsymbol{f}^{\prime}\right|=\left|\boldsymbol{g}^{\prime}\right|, \\
\boldsymbol{c}^{\prime} \cdot \boldsymbol{f}=\boldsymbol{d}^{\prime} \cdot \boldsymbol{g}, \quad \text { and } \quad \boldsymbol{c}^{\prime} \cdot \boldsymbol{f}^{\prime}=\boldsymbol{d}^{\prime} \cdot \boldsymbol{g}^{\prime} .
\end{array}\right\}
$$

Notice from (19) 1 and $(24)_{4,5}$ that

$$
d^{\prime \prime} \cdot g=0
$$


If we set $\boldsymbol{t}=\boldsymbol{d}^{\prime}$, we observe that

$$
n=\frac{t \times g}{|t \times g|}
$$

is a unit vector orthogonal to $\mathcal{S}$ along its directrix $\mathcal{C}_{S}$, and that

$$
m=n \times t
$$

is a unit vector that is tangent to $\mathcal{S}$ and normal to $\mathcal{C}_{s}$. The triad $\{\boldsymbol{t}, \boldsymbol{m}, \boldsymbol{n}\}$ is a Darboux frame for $\mathcal{C}_{s}$, from which it follows that

$$
\boldsymbol{t}^{\prime}=\kappa_{g} \boldsymbol{m}+\kappa_{n} \boldsymbol{n}, \quad \boldsymbol{m}^{\prime}=-\kappa_{g} \boldsymbol{t}+\tau_{g} \boldsymbol{n}, \quad \text { and } \quad \boldsymbol{n}^{\prime}=-\kappa_{n} \boldsymbol{t}-\tau_{g} \boldsymbol{m},
$$

where $\kappa_{g}, \kappa_{n}$, and $\tau_{g}$, respectively, denote the geodesic curvature, normal curvature, and geodesic torsion of $\mathcal{C}_{s}$. Since the referential directrix $\mathcal{C}_{r}$ consists of three straightline segments and $\chi$ is an isometric deformation, $\mathcal{C}_{s}$ is the union of three geodesic curves. Thus, the geodesic curvature of $\mathcal{C}_{S}$ obeys

$$
\kappa_{g}=0
$$

except at $b$ and $\ell+b$, where it is undefined. From $(24)_{4}$, we see that the angle between $\boldsymbol{t}$ and $\boldsymbol{g}$ must be identical to the angle $\theta$ between $\boldsymbol{c}^{\prime}$ and $\boldsymbol{f}$, and thus, that $\boldsymbol{g}$ admits the representation

$$
\boldsymbol{g}=\cos \theta \boldsymbol{t}+\sin \theta \boldsymbol{m} .
$$

Moreover, we find from (28)-(30) that

$$
\boldsymbol{g}^{\prime}=-\theta^{\prime}(\sin \theta \boldsymbol{t}-\cos \theta \boldsymbol{m})+\left(\kappa_{n} \cos \theta+\tau_{g} \sin \theta\right) \boldsymbol{n} .
$$

Using (31) and (18) in $(24)_{3}$, we arrive at the requirement

$$
\kappa_{n} \cos \theta+\tau_{g} \sin \theta=0
$$

from which we see that (31) simplifies to

$$
\boldsymbol{g}^{\prime}=-\theta^{\prime}(\sin \theta \boldsymbol{t}-\cos \theta \boldsymbol{m}) .
$$

Moreover, we find from $(28)_{2},(29)$, and (32) that

$$
\boldsymbol{m}^{\prime}=-\kappa_{n} \cot \theta \boldsymbol{n},
$$

and from (28) 3 and (32), that

$$
\boldsymbol{n}^{\prime}=-\kappa_{n}(\boldsymbol{t}-\cot \theta \boldsymbol{m})
$$


Since $\boldsymbol{c}(\alpha)+\beta_{-}(\alpha) \boldsymbol{f}(\alpha)$ and $\boldsymbol{c}(\alpha)+\beta_{+}(\alpha) \boldsymbol{f}(\alpha)$ are the end points of the ruling that passes through $c(\alpha)$, we find that it is possible to determine explicit representations for the functions $\beta_{ \pm}$entering the definition (20) of $\mathcal{P}$. To express those representations, we first let $\alpha_{L} \in[b, \ell+b]$ denote the largest value of $\alpha$ for which the ruling containing $\boldsymbol{c}\left(\alpha_{L}\right)$ intersects the left-hand edge $\partial \mathcal{D}_{L}$ of $\mathcal{D}$ and let $\alpha_{R} \in[b, \ell+b]$ denote the smallest value of $\alpha$ for which the ruling containing $c\left(\alpha_{R}\right)$ intersects the right-hand edge $\partial \mathcal{D}_{R}$ of $\mathcal{D}$, as depicted in Fig. 1. Notice that these values of $\alpha$ must satisfy $\alpha_{L}<\alpha_{R}$. If $\mathcal{S}$ is orientable, we find that

$$
\beta_{-}(\alpha)= \begin{cases}0, & \alpha \in[0, b), \\ (b-\alpha) \sec \theta(\alpha), & \alpha \in\left[b, \alpha_{L}\right), \\ -b \csc \theta(\alpha), & \alpha \in\left[\alpha_{L}, \ell+b\right), \\ (\ell+2 b-\alpha) \sec \theta(\alpha), & \alpha \in[\ell+b, \ell+2 b],\end{cases}
$$

and that

$$
\beta_{+}(\alpha)= \begin{cases}-\alpha \sec \theta(\alpha), & \alpha \in[0, b), \\ b \csc \theta(\alpha), & \alpha \in\left[b, \alpha_{R}\right), \\ (\ell+b-\alpha) \sec \theta(\alpha), & \alpha \in\left[\alpha_{R}, \ell+b\right), \\ 0, & \alpha \in[\ell+b, \ell+2 b] .\end{cases}
$$

If, alternatively, $\mathcal{S}$ is nonorientable, we find that

$$
\beta_{-}(\alpha)= \begin{cases}\alpha \sec \theta(\alpha), & \alpha \in[0, b), \\ -b \csc \theta(\alpha), & \alpha \in[b, \ell+b), \\ (\ell+2 b-\alpha) \sec \theta(\alpha), & \alpha \in[\ell+b, \ell+2 b],\end{cases}
$$

and that

$$
\beta_{+}(\alpha)= \begin{cases}0, & \alpha \in[0, b), \\ (b-\alpha) \sec \theta(\alpha), & \alpha \in\left[b, \alpha_{L}\right), \\ b \csc \theta(\alpha), & \alpha \in\left[\alpha_{L}, \alpha_{R}\right), \\ (\ell+b-\alpha) \sec \theta(\alpha), & \alpha \in\left[\alpha_{R}, \ell+b\right), \\ 0, & \alpha \in[\ell+b, \ell+2 b] .\end{cases}
$$

\subsection{Natural Extension of the Parameterization of $\mathcal{S}$}

From (22), we notice that the function $\hat{\boldsymbol{r}}: \mathcal{P} \rightarrow \mathcal{S}$ defined in (22) possesses a natural extension to the set

$$
\mathcal{P}_{e}=\left\{(\alpha, \beta) \in \mathbb{R}^{2} \mid \alpha \in[0, \ell+2 b], \beta \in \mathbb{R}\right\}
$$


When using that extension, it is important, however, to keep in mind that $\hat{\boldsymbol{r}}(\alpha, \beta)$ need not be a point on $\mathcal{S}$ unless $(\alpha, \beta)$ is an element of $\mathcal{P}$.

Defining parameter sets

$$
\left.\begin{array}{l}
\mathcal{I}=\left\{\alpha \in \mathbb{R} \mid \alpha \in\left[b, \alpha_{L}\right] \cup\left[\alpha_{R}, \ell+b\right]\right\}, \\
\mathcal{K}=\{\alpha \in \mathbb{R} \mid \alpha \in[0, b] \cup[\ell+b, \ell+2 b]\}
\end{array}\right\}
$$

we observe that the rulings that intersect the left- and right-hand edges $\partial \mathcal{D}_{L}$ and $\partial \mathcal{D}_{R}$ of $\mathcal{D}$ induce a bijection $\hat{\alpha}: \mathcal{I} \rightarrow \mathcal{K}$. Moreover, we see that the bijection $\hat{\alpha}: \mathcal{I} \rightarrow \mathcal{K}$ can be used to express a constraint on $\hat{\boldsymbol{r}}: \mathcal{P}_{e} \rightarrow \mathcal{S}$. The specific forms of the bijection and the constraint on the extension of $\hat{\boldsymbol{r}}$ differ depending on the orientability of $\mathcal{S}$.

\subsubsection{Orientable Closed Ribbons}

For an orientable ribbon, the bijection $\hat{\alpha}: \mathcal{I} \rightarrow \mathcal{K}$ takes the form

$$
\hat{\alpha}(\alpha)= \begin{cases}b+\ell+(\alpha-b) \tan \theta(\alpha), & \alpha \in\left[b, \alpha_{L}\right], \\ b+(\alpha-b-\ell) \tan \theta(\alpha), & \alpha \in\left[\alpha_{R}, \ell+b\right],\end{cases}
$$

as exemplified in Fig. 1a.

Recalling that, for connecting edge conditions (10) leading to an orientable closed ribbon $\mathcal{S}$, the deformed images of the referential rulings that terminate at the points $x_{2} \boldsymbol{l}_{2}$ and $\ell \boldsymbol{t}_{1}+x_{2} \boldsymbol{\iota}_{2}$ on the left- and right-hand edges $\partial \mathcal{D}_{L}$ and $\partial \mathcal{D}_{R}$ of the strip $\mathcal{D}$ must, for each $x_{2} \in[0,2 b]$, meet to form a single ruling that contains the point $\chi\left(0, x_{2}, 0\right)=\chi\left(\ell, x_{2}, 0\right)$ on $\mathcal{S}$, we obtain conditions that $\hat{\boldsymbol{r}}: \mathcal{P}_{e} \rightarrow \mathcal{S}$ must satisfy for each $\alpha \in\left[b, \alpha_{L}\right]$ and for each $\alpha \in\left[\alpha_{R}, \ell+b\right]$ :

- For $\alpha \in\left[b, \alpha_{L}\right]$,

$$
\hat{\boldsymbol{r}}\left(\alpha, \beta_{-}(\alpha)+\beta\right)=\hat{\boldsymbol{r}}(\hat{\alpha}(\alpha), \beta), \quad \beta \in\left[\beta_{-}(\hat{\alpha}(\alpha)), 0\right]
$$

- For $\alpha \in\left[\alpha_{R}, \ell+b\right]$,

$$
\hat{\boldsymbol{r}}\left(\alpha, \beta_{+}(\alpha)+\beta\right)=\hat{\boldsymbol{r}}(\hat{\alpha}(\alpha), \beta), \quad \beta \in\left[0, \beta_{+}(\hat{\alpha}(\alpha))\right]
$$

Computing partial derivatives of $\hat{\boldsymbol{r}}$ with respect to the parameters $\alpha$ and $\beta$ on both sides of (43), we see that, for each $\alpha \in\left[b, \alpha_{L}\right]$, 


$$
\begin{aligned}
& \left|\hat{\boldsymbol{r}}_{\alpha}(\hat{\alpha}(\alpha), \beta) \times \hat{\boldsymbol{r}}_{\beta}(\hat{\alpha}(\alpha), \beta)\right|\left|\hat{\alpha}^{\prime}(\alpha)\right| \\
& \quad=\left|\hat{\boldsymbol{r}}_{\alpha}\left(\alpha, \beta_{-}(\alpha)+\beta\right) \times \hat{\boldsymbol{r}}_{\beta}\left(\alpha, \beta_{-}(\alpha)+\beta\right)\right|, \quad \beta \in\left[\beta_{-}(\hat{\alpha}(\alpha)), 0\right] .
\end{aligned}
$$

Performing analogous calculations on both sides of (44), we see that, for $\alpha \in\left[\alpha_{R}, \ell+\right.$ b],

$$
\begin{aligned}
& \left|\hat{\boldsymbol{r}}_{\alpha}(\hat{\alpha}(\alpha), \beta) \times \hat{\boldsymbol{r}}_{\beta}(\hat{\alpha}(\alpha), \beta)\right|\left|\hat{\alpha}^{\prime}(\alpha)\right| \\
& \quad=\left|\hat{\boldsymbol{r}}_{\alpha}\left(\alpha, \beta_{+}(\alpha)+\beta\right) \times \hat{\boldsymbol{r}}_{\beta}\left(\alpha, \beta_{+}(\alpha)+\beta\right)\right|, \quad \beta \in\left[0, \beta_{+}(\hat{\alpha}(\alpha))\right] .
\end{aligned}
$$

Invoking (36), (37), (44), and (46) followed by the change of variables $(\hat{\alpha}(\alpha), \beta) \mapsto$ $\left(\alpha, \beta_{+}(\alpha)+\beta\right)$, we find that, for any integrable function $f: \mathcal{S} \rightarrow \mathbb{R}$,

$$
\begin{aligned}
\int_{0}^{b} \int_{\beta_{-}(\alpha)}^{\beta_{+}(\alpha)}(f \circ \hat{\boldsymbol{r}})\left|\hat{\boldsymbol{r}}_{\alpha} \times \hat{\boldsymbol{r}}_{\beta}\right| \mathrm{d} \beta \mathrm{d} \alpha & =\int_{\alpha_{R}}^{\ell+b} \int_{\beta_{+}(\alpha)+\beta_{-}(\hat{\alpha}(\alpha))}^{\beta_{+}(\alpha)+\beta_{+}(\hat{\alpha}(\alpha))}(f \circ \hat{\boldsymbol{r}})\left|\hat{\boldsymbol{r}}_{\alpha} \times \hat{\boldsymbol{r}}_{\beta}\right| \mathrm{d} \beta \mathrm{d} \alpha \\
& =\int_{\alpha_{R}}^{\ell+b} \int_{\beta_{+}(\alpha)}^{b \csc \theta(\alpha)}(f \circ \hat{\boldsymbol{r}})\left|\hat{\boldsymbol{r}}_{\alpha} \times \hat{\boldsymbol{r}}_{\beta}\right| \mathrm{d} \beta \mathrm{d} \alpha .
\end{aligned}
$$

Hence, invoking (36) once again, we obtain the identity

$$
\begin{array}{r}
\left(\int_{0}^{b}+\int_{\alpha_{R}}^{\ell+b}\right) \int_{\beta_{-}(\alpha)}^{\beta_{+}(\alpha)}(f \circ \hat{\boldsymbol{r}})\left|\hat{\boldsymbol{r}}_{\alpha} \times \hat{\boldsymbol{r}}_{\beta}\right| \mathrm{d} \beta \mathrm{d} \alpha \\
\quad=\int_{\alpha_{R}}^{\ell+b} \int_{-b \csc \theta(\alpha)}^{b \csc \theta(\alpha)}(f \circ \hat{\boldsymbol{r}})\left|\hat{\boldsymbol{r}}_{\alpha} \times \hat{\boldsymbol{r}}_{\beta}\right| \mathrm{d} \beta \mathrm{d} \alpha .
\end{array}
$$

Similarly, invoking (38), (39), (43), and (45) followed by the change of variables $(\hat{\alpha}(\alpha), \beta) \mapsto\left(\alpha, \beta_{-}(\alpha)+\beta\right)$, we obtain the identity

$$
\begin{gathered}
\left(\int_{\ell+b}^{\ell+2 b}+\int_{b}^{\alpha_{L}}\right) \int_{\beta_{-}(\alpha)}^{\beta_{+}(\alpha)}(f \circ \hat{\boldsymbol{r}})\left|\hat{\boldsymbol{r}}_{\alpha} \times \hat{\boldsymbol{r}}_{\beta}\right| \mathrm{d} \beta \mathrm{d} \alpha \\
=\int_{b}^{\alpha_{L}} \int_{-b \csc \theta(\alpha)}^{b \csc \theta(\alpha)}(f \circ \hat{\boldsymbol{r}})\left|\hat{\boldsymbol{r}}_{\alpha} \times \hat{\boldsymbol{r}}_{\beta}\right| \mathrm{d} \beta \mathrm{d} \alpha .
\end{gathered}
$$

\subsubsection{Nonorientable Closed Ribbons}

For a nonorientable ribbon, the bijection $\hat{\alpha}$ takes the form

$$
\hat{\alpha}(\alpha)= \begin{cases}b+(\alpha-\ell-b) \tan \theta(\alpha), & \alpha \in\left[b, \alpha_{L}\right], \\ \ell+b+(b-\alpha) \tan \theta(\alpha), & \alpha \in\left[\alpha_{R}, \ell+b\right],\end{cases}
$$

as depicted in Fig. $1 b$.

Recalling that, for connecting edge conditions of type (11) leading to a nonorientable closed ribbon $\mathcal{S}$, the deformed images of the referential rulings that terminate 
at the points $x_{2} \boldsymbol{l}_{2}$ and $\ell \boldsymbol{t}_{1}+\left(2 b-x_{2}\right) \boldsymbol{\iota}_{2}$ on the left- and right-hand edges $\partial \mathcal{D}_{L}$ and $\partial \mathcal{D}_{R}$ of the strip $\mathcal{D}$ must, for each $x_{2} \in[0,2 b]$, meet to form a single ruling on $\mathcal{S}$ that contains the point $\chi\left(0, x_{2}, 0\right)=\chi\left(\ell, 2 b-x_{2}, 0\right)$, we obtain a condition that $\hat{\boldsymbol{r}}: \mathcal{P}_{e} \rightarrow \mathcal{S}$ must satisfy for each $\alpha \in\left[b, \alpha_{L}\right] \cup\left[\alpha_{R}, \ell+b\right]:$

$$
\hat{\boldsymbol{r}}\left(\alpha, \beta_{+}(\alpha)-\beta\right)=\hat{\boldsymbol{r}}(\hat{\alpha}(\alpha), \beta), \quad \beta \in\left[\beta_{-}(\hat{\alpha}(\alpha)), 0\right] .
$$

From calculations analogous to those leading to (45) and (46), we find from (51) that, for each $\alpha \in\left[b, \alpha_{L}\right] \cup\left[\alpha_{R}, \ell+b\right]$,

$$
\begin{aligned}
& \left|\hat{\boldsymbol{r}}_{\alpha}(\hat{\alpha}(\alpha), \beta) \times \hat{\boldsymbol{r}}_{\beta}(\hat{\alpha}(\alpha), \beta)\right|\left|\hat{\alpha}^{\prime}(\alpha)\right| \\
& \quad=\left|\hat{\boldsymbol{r}}_{\alpha}\left(\alpha, \beta_{+}(\alpha)-\beta\right) \times \hat{\boldsymbol{r}}_{\beta}\left(\alpha, \beta_{+}(\alpha)-\beta\right)\right|, \quad \beta \in\left[\beta_{-}(\hat{\alpha}(\alpha)), 0\right] .
\end{aligned}
$$

Finally, invoking (38), (39), (51), and (52), followed by the change of variables $(\hat{\alpha}(\alpha), \beta) \mapsto\left(\beta_{+}(\alpha)-\beta\right)$, we find that the identities (48) and (49) also hold if $\mathcal{S}$ is nonorientable.

\subsection{Consequences of Requiring that Rulings Do Not Overlap}

It is not generally possible to ensure that the rulings do not intersect in the interior of $\mathcal{S}$ without placing a suitable restriction on $\theta$. To determine such a restriction, choose $\alpha_{1}$ and $\alpha_{2}$ satisfying $b \leq \alpha_{1} \leq \alpha_{2} \leq \ell+b$. The points $\boldsymbol{d}\left(\alpha_{1}\right)+\beta_{+}\left(\alpha_{1}\right) \boldsymbol{g}\left(\alpha_{1}\right)$ and $\boldsymbol{d}\left(\alpha_{2}\right)+\beta_{+}\left(\alpha_{2}\right) \boldsymbol{g}\left(\alpha_{2}\right)$ are on the edge of the ribbon and are obtained by starting at the points $\boldsymbol{d}\left(\alpha_{1}\right)$ and $\boldsymbol{d}\left(\alpha_{2}\right)$ and proceeding along the associated rulings in the direction of $\boldsymbol{g}$. Consider the geodesic that passes through each of these points and is orthogonal to the midline of the ribbon. Those geodesics intersect the midline at the points corresponding to the values $\alpha_{1}+b \cot \theta\left(\alpha_{1}\right)$ and $\alpha_{2}+b \cot \theta\left(\alpha_{2}\right)$ of $\alpha$. Since the rulings do not intersect in the interior of the ribbon, we must have

$$
\alpha_{1}+b \cot \theta\left(\alpha_{1}\right) \leq \alpha_{2}+b \cot \theta\left(\alpha_{2}\right)
$$

Moving all the terms to the right-hand side of the inequality (53), dividing by $\alpha_{2}-\alpha_{1}$, and taking the limit as $\alpha_{2}$ goes to $\alpha_{1}$, we find that

$$
0 \leq 1-b \csc ^{2} \theta\left(\alpha_{1}\right) \theta^{\prime}\left(\alpha_{1}\right)
$$

Similarly, considering the points $\boldsymbol{d}\left(\alpha_{1}\right)+\beta_{-}\left(\alpha_{1}\right) \boldsymbol{g}\left(\alpha_{1}\right)$ and $\boldsymbol{d}\left(\alpha_{2}\right)+\beta_{-}\left(\alpha_{2}\right) \boldsymbol{g}\left(\alpha_{2}\right)$ on the edge of the ribbon obtained by starting at the points $\boldsymbol{d}\left(\alpha_{1}\right)$ and $\boldsymbol{d}\left(\alpha_{2}\right)$, proceeding along the associated ruling in the direction of $-\boldsymbol{g}$, and taking steps analogous to those leading to (54), we find that

$$
0 \leq 1+b \csc ^{2} \theta\left(\alpha_{1}\right) \theta^{\prime}\left(\alpha_{1}\right)
$$


Since $\csc \theta>0$, we may express the conditions (54) and (55), which must hold for all $\alpha_{1}$, in the form

$$
0 \leq \sin ^{2} \theta \pm b \theta^{\prime}
$$

Recognizing that $\sin ^{2} \theta+b \theta^{\prime}$ and $\sin ^{2} \theta-b \theta^{\prime}$ cannot be simultaneously negative, we deduce that the inequalities in (56) are equivalent to the single inequality

$$
0 \leq\left(\sin ^{2} \theta-b \theta^{\prime}\right)\left(\sin ^{2} \theta+b \theta^{\prime}\right)=\sin ^{4} \theta-b^{2} \theta^{\prime 2} .
$$

For situations in which the directrix is a line of curvature, a condition equivalent to (57) was derived by Hornung (2011c) and was shown to imply that, in the interior of the ribbon, rulings cannot intersect locally. ${ }^{4}$

\section{Bending Energy}

The energy stored during an isometric deformation of the unstretchable rectangular strip $\mathcal{D}$ to a closed ribbon $\mathcal{S}$ is due purely to bending, and thus, the curvature tensor of $\mathcal{S}$ serves as a measure of strain. For simplicity, we assume that the bending energy density depends quadratically on the curvature tensor and is isotropic. Since the Gaussian curvature vanishes identically for any isometric deformation $\chi: \mathcal{D} \rightarrow \mathcal{S}$, it follows that the total bending energy $E$ of $\mathcal{S}$ must be of the simple form

$$
E=2 \mu \int_{\mathcal{S}} H^{2} \mathrm{~d} a,
$$

with $\mu>0$ being a material constant known as the bending modulus.

\subsection{Consequences of Requiring that the Total Bending Energy Be Bounded}

It is possible for an isometric deformation $\chi$ of $\mathcal{D}$ to result in a surface $\mathcal{S}$ with unbounded bending energy $E$. This can occur if, for example, a collection of rulings that meet at a point on $\mathcal{D}$ cover a curved portion of $\mathcal{S}$. To verify this assertion, consider a portion of the surface $\mathcal{T}_{c}$ covered by rulings that intersect on the boundary of $\mathcal{S}$ as discussed in Sect. 2.3. Let $\phi_{1}$ and $\phi_{2}$ be the minimum and maximum angles associated with the referential preimage $\mathcal{T}_{r}$ of $\mathcal{T}_{c}$. With reference to the parameterization (12), we find that the mean curvature $H$ of $\mathcal{T}_{c}$ can be expressed as

$$
H(\tilde{\boldsymbol{r}}(\phi, \beta))=\frac{\boldsymbol{n}(\phi) \cdot \boldsymbol{g}^{\prime \prime}(\phi)}{2 \beta},
$$

\footnotetext{
${ }^{4}$ This means that there is a neighborhood of each $\alpha \in[b, \ell+b]$ such that, for any choices $\alpha_{1}$ and $\alpha_{2}$, $\alpha_{1} \neq \alpha_{2}$ in that neighborhood, the rulings along $\boldsymbol{g}\left(\alpha_{1}\right)$ and $\boldsymbol{g}\left(\alpha_{2}\right)$ do not intersect. 
and thus, that the bending energy of $\mathcal{T}_{c}$ is given by

$$
2 \mu \int_{\mathcal{T}_{c}} H^{2} \mathrm{~d} a=2 \mu \int_{\phi_{1}}^{\phi_{2}} \int_{0}^{2 b \csc \phi} \frac{\left(\boldsymbol{n}(\phi) \cdot \boldsymbol{g}^{\prime \prime}(\phi)\right)^{2}}{4 \beta} \mathrm{d} \beta \mathrm{d} \phi,
$$

which is unbounded unless $H=0$. From the foregoing argument, we infer that the bending energy $E$ of $\mathcal{S}$ cannot be bounded unless each portion of $\mathcal{S}$ between a pair of rulings that intersect at a point on $\partial \mathcal{S}$ is flat.

We next show that if $\chi$ is $C^{2}$ on the interior of $\mathcal{D}$ and the bending energy $E$ of $\mathcal{S}=\chi(\mathcal{D})$ is bounded, then $\chi$ must be $C^{1}$ on the entirety of $\partial \mathcal{D}$. To establish this claim, we first collect some preliminary results. Recall that in Sect. 2.2 we argued that $\mathcal{D}$ can be completely covered by a continuous family of rulings. This means that there is a function $\hat{f}$ defined on the interior of $\mathcal{D}$ with unit vector values and with the property that $\hat{\boldsymbol{f}}(\boldsymbol{x})$ is parallel to the ruling that passes through $\boldsymbol{x}$. Let $\hat{\boldsymbol{f}}_{\perp}$ be the unit vector chosen such that it is orthogonal to $\hat{f}$ and such that $\left(\hat{f} \times \hat{f}_{\perp}\right) \cdot \boldsymbol{\iota}_{3}>0$. Then, using $Q=\nabla \chi$ to denote the gradient of $\chi$, we see that the vectors

$$
\hat{g}=Q \hat{f} \quad \text { and } \quad \hat{g}_{\perp}=Q \hat{f}_{\perp}
$$

must be tangent to $\mathcal{S}$, with $\hat{\boldsymbol{g}}(\boldsymbol{x})$ being parallel to the ruling on $\mathcal{S}$ that passes through $\chi(\boldsymbol{x})$. Since the $\chi$ is an isometric deformation, $|\boldsymbol{Q} \hat{\boldsymbol{f}}|=|\hat{\boldsymbol{f}}|=1$ and $\left|\boldsymbol{Q} \hat{\boldsymbol{f}}_{\perp}\right|=\left|\hat{\boldsymbol{f}}_{\perp}\right|=$ 1 and we see from (61) that $\hat{\boldsymbol{g}}(\boldsymbol{x})$ and $\hat{\boldsymbol{g}}_{\perp}(\boldsymbol{x})$ are of unit magnitude. From (61), we see further that $Q$ admits the representation

$$
Q=\hat{g} \otimes \hat{f}+\hat{\boldsymbol{g}}_{\perp} \otimes \hat{\boldsymbol{f}}_{\perp}
$$

Since $\hat{\boldsymbol{f}}, \hat{\boldsymbol{f}}_{\perp}, \hat{\boldsymbol{g}}$, and $\hat{\boldsymbol{g}}_{\perp}$ are uniform along each ruling, we deduce from (62) that $\boldsymbol{Q}$ must also be uniform along each ruling.

Consider a point $\boldsymbol{x}_{\circ}$ on $\partial \mathcal{D}$ where rulings intersect, so that the corresponding portion of $\mathcal{S}$ is flat. If $\mathcal{T}_{r}$ denotes the corresponding triangular region in $\mathcal{D}$ that is covered by the rulings that intersect at $\boldsymbol{x}_{\circ}$, then $\boldsymbol{Q}$ is uniform on $\mathcal{T}_{r}$ since $\chi\left(\mathcal{T}_{r}\right)$ is flat. Thus, writing $Q_{\circ}$ for the value of $Q$ on $\mathcal{T}_{r}$ and invoking the continuity of $\chi$, we find that

$$
\chi\left(x_{\circ}\right)=\chi\left(x_{f}\right)+Q_{\circ}\left(x_{\circ}-x_{f}\right)
$$

for any point $\boldsymbol{x}_{f} \in \mathcal{T}_{r}$. Moreover, for any point $\boldsymbol{x} \in \mathcal{D}$ sufficiently close to $\boldsymbol{x}_{\circ}$, choose $\boldsymbol{x}_{f} \in \mathcal{T}_{r}$ such that $\left|\boldsymbol{x}-\boldsymbol{x}_{f}\right|=2\left|\boldsymbol{x}-\boldsymbol{x}_{\circ}\right|$. We also have the relation

$$
\chi(x)-\chi\left(x_{f}\right)=\int_{0}^{1} Q\left(x_{f}+t\left(x-x_{f}\right)\right)\left(x-x_{f}\right) \mathrm{d} t .
$$

Notice that $\left|\tilde{\boldsymbol{x}}-\boldsymbol{x}_{f}\right| \rightarrow 0$ as $\boldsymbol{x} \rightarrow \boldsymbol{x}_{\circ}$. Then, for $\varepsilon>0$ and sufficiently small, there is a compact subset $\mathcal{D}_{\varepsilon}$ of the interior of $\mathcal{D}$ such that any ruling that intersects the set

$$
\left\{x \in \mathcal{D}|| x-x_{\circ} \mid<\varepsilon\right\}
$$


also intersects $\mathcal{D}_{\varepsilon}$. Since $\mathcal{D}_{\varepsilon}$ is compact and $\boldsymbol{Q}$ is continuous, $\boldsymbol{Q}$ is uniformly continuous on $\mathcal{D}_{\varepsilon}$; moreover, since $\boldsymbol{Q}$ is uniform along each ruling, it must be uniformly continuous on a set of the form

$$
\left\{x \in \mathcal{D}|| x-x_{\circ} \mid<\eta\right\}
$$

for some $\eta>0$. From this, (63), and (64), we thus see that

$$
\begin{aligned}
\lim _{x \rightarrow x_{\circ}} & \frac{\left|\chi(x)-\chi\left(x_{\circ}\right)-Q_{\circ}\left(x-x_{\circ}\right)\right|}{2\left|x-x_{\circ}\right|} \\
& =\lim _{x \rightarrow x_{\circ}}\left|\left(\int_{0}^{1} \boldsymbol{Q}\left(x_{f}+t\left(x-x_{f}\right)\right) \mathrm{d} t-\boldsymbol{Q}\left(\boldsymbol{x}_{f}\right)\right) \frac{\boldsymbol{x}-\boldsymbol{x}_{f}}{\left|\boldsymbol{x}-\boldsymbol{x}_{f}\right|}\right| \\
& \leq \lim _{x \rightarrow x_{\circ}} \int_{0}^{1}\left|\boldsymbol{Q}\left(\boldsymbol{x}_{f}+t\left(\boldsymbol{x}-\boldsymbol{x}_{f}\right)\right)-\boldsymbol{Q}\left(\boldsymbol{x}_{f}\right)\right| \mathrm{d} t \\
& =0
\end{aligned}
$$

from which we deduce that $\nabla \chi\left(x_{\circ}\right)=Q_{\circ}$, and, thus, that, as claimed, $\chi$ is $C^{1}$ at $\boldsymbol{x}_{\circ}$.

\subsection{Dimensionally Reduced Bending Energy}

Using the parameterization $\hat{\boldsymbol{r}}$ of $\mathcal{S}$ from (22), we may express the bending energy $E$ defined in (58) in the form

$$
E=2 \mu \int_{0}^{\ell+2 b} \int_{\beta_{-}(\alpha)}^{\beta_{+}(\alpha)}(H \circ \hat{\boldsymbol{r}})^{2}\left|\hat{\boldsymbol{r}}_{\alpha} \times \hat{\boldsymbol{r}}_{\beta}\right| \mathrm{d} \beta \mathrm{d} \alpha
$$

To reduce $E$ to an integral over the spatial directrix $\mathcal{C}_{s}$, we first use the decomposition

$$
[0, \ell+2 b]=[0, b] \cup\left[b, \alpha_{L}\right] \cup\left[\alpha_{L}, \alpha_{R}\right] \cup\left[\alpha_{R}, \ell+b\right] \cup[\ell+b, \ell+2 b]
$$

of $[0, \ell+2 b]$ to rewrite $(67)$ as

$$
\begin{aligned}
E=2 \mu\left(\int_{0}^{b}\right. & +\int_{b}^{\alpha_{L}}+\int_{\alpha_{L}}^{\alpha_{R}}+\int_{\alpha_{R}}^{\ell+b} \\
& \left.+\int_{\ell+b}^{\ell+2 b}\right) \int_{\beta_{-}(\alpha)}^{\beta_{+}(\alpha)}(H \circ \hat{\boldsymbol{r}})^{2}\left|\hat{\boldsymbol{r}}_{\alpha} \times \hat{\boldsymbol{r}}_{\beta}\right| \mathrm{d} \beta \mathrm{d} \alpha .
\end{aligned}
$$

Next, utilizing (48), (49), and (37)-(38), we find that (69) can be rewritten as

$$
E=2 \mu\left(\int_{b}^{\alpha_{L}}+\int_{\alpha_{L}}^{\alpha_{R}}+\int_{\alpha_{R}}^{\ell+b}\right) \int_{-b \csc \theta(\alpha)}^{b \csc \theta(\alpha)}(H \circ \hat{\boldsymbol{r}})^{2}\left|\hat{\boldsymbol{r}}_{\alpha} \times \hat{\boldsymbol{r}}_{\beta}\right| \mathrm{d} \beta \mathrm{d} \alpha
$$


from which we see that

$$
E=2 \mu \int_{b}^{\ell+b} \int_{-b \csc \theta(\alpha)}^{b \csc \theta(\alpha)}(H \circ \hat{\boldsymbol{r}})^{2}\left|\hat{\boldsymbol{r}}_{\alpha} \times \hat{\boldsymbol{r}}_{\beta}\right| \mathrm{d} \beta \mathrm{d} \alpha .
$$

Finally, noting from (22), (33), and (35) that

$$
2(H \circ \hat{\boldsymbol{r}})^{2}\left|\hat{\boldsymbol{r}}_{\alpha} \times \hat{\boldsymbol{r}}_{\beta}\right|=\frac{\left(\boldsymbol{n}_{\alpha} \cdot \hat{\boldsymbol{r}}_{\alpha}\right)^{2}}{2\left|\hat{\boldsymbol{r}}_{\alpha} \times \hat{\boldsymbol{r}}_{\beta}\right|^{3}}=\frac{\left|\boldsymbol{d}^{\prime \prime}\right|^{2}}{2 \sin ^{2} \theta\left(\sin \theta-\beta \theta^{\prime}\right)},
$$

where $\sin \theta-\beta \theta^{\prime}$ must be positive to ensure that rulings do not intersect in the interior of $\mathcal{S}$. We infer that the innermost integral of (71) can be evaluated in closed form, and thus, that the bending energy $E$ of $\mathcal{S}$ can be expressed as a single integral from $\alpha=b$ to $\alpha=\ell+b$ :

$$
E=\frac{\mu}{2} \int_{b}^{\ell+b} \frac{\left|\boldsymbol{d}^{\prime \prime}\right|^{2}}{\theta^{\prime} \sin ^{2} \theta} \log \frac{\sin ^{2} \theta+b \theta^{\prime}}{\sin ^{2} \theta-b \theta^{\prime}} \mathrm{d} \alpha
$$

The term $\sin ^{2} \theta+b \theta^{\prime}$ must also be positive to ensure that rulings do not intersect in the interior of $\mathcal{S}$, and thus, that the argument of the logarithm in (73) is always positive. The dimensional reduction (73) is valid for orientable and nonorientable closed ribbons since the identities (48) and (49) needed to pass from (69) to (71) hold regardless of the orientability of $\mathcal{S}$, as noted at the end of Sect. 2 .

When using $E$ in the form (73), it is essential to keep in mind that $\theta^{\prime}$ is not defined if $\boldsymbol{d}^{\prime \prime}$ vanishes or does not exist. In addition, the bending energy density appearing in (73) is not defined if $\theta^{\prime}$ vanishes. Using the asymptotic properties of the logarithm, we find, however, that

$$
\lim _{\theta^{\prime} \rightarrow 0} \frac{\left|\boldsymbol{d}^{\prime \prime}\right|^{2}}{\theta^{\prime} \sin ^{2} \theta} \log \frac{\sin ^{2} \theta+b \theta^{\prime}}{\sin ^{2} \theta-b \theta^{\prime}}=\frac{2 b\left|\boldsymbol{d}^{\prime \prime}\right|^{2}}{\sin ^{4} \theta} .
$$

The expression on the right-hand side of (74) is the bending energy density that arises from using the dimensional reduction if the rulings are locally parallel, in which case $\theta^{\prime}=0$. Hence, the bending energy density appearing in (73) can be used even if $\theta^{\prime}$ vanishes with the understanding that it is continuous at any point of the directrix where that occurs.

\subsection{Wunderlich Formula for the Bending Energy}

Wunderlich (1962) was the first to carry out the dimensional reduction in the bending energy (58) for the problem of isometrically deforming an unstretchable rectangular strip $\mathcal{D}$ of length $\ell$ and finite width $2 b$ to a Möbius band $\mathcal{S}$ with a single half-twist, 
leading to the expression ${ }^{5}$

$$
E_{W}=\frac{\mu}{2} \int_{b}^{\ell+b} \bar{\kappa}_{0}^{2} V \log \frac{V+b}{V-b} \mathrm{~d} \alpha
$$

involving integration over the midline $\mathcal{M}$ of $\mathcal{S}$, where $\bar{\kappa}_{0}$ is the principal normal curvature of $\mathcal{M}$, as parameterized by (17), and $V$ is defined by

$$
V=\frac{\sin ^{2} \theta}{\theta^{\prime}},
$$

where $\theta$ denotes the angle between the tangent and generators of $\mathcal{M}$. Invoking Euler's formula ${ }^{6}$ for the curvature $\kappa=\left|\boldsymbol{d}^{\prime \prime}\right|$, we see that the principal normal curvature of $\mathcal{M}$ can be expressed as

$$
\bar{\kappa}_{0}=\frac{\left|\boldsymbol{d}^{\prime \prime}\right|}{\sin ^{2} \theta} .
$$

Using (76) and (77) in (75), we find that (75) is equivalent to (73).

\section{Ribbons with Piecewise Continuous Curvature}

Although we derived the dimensionally reduced bending energy (73) under the assumption that the deformation $\chi$ is $C^{2}$, and hence, that the curvature of the closed ribbon $\mathcal{S}$ is continuous, (73) is well-defined and finite under weaker regularity conditions. To specify such conditions, it is first essential to select the independent variables over which (73) is to be minimized. From the structure of (73), it might seem natural to view (73) as a functional of $\boldsymbol{d}$ and $\theta$. Recalling from (30) that $\cos \theta=\boldsymbol{d}^{\prime} \cdot \boldsymbol{g}$ and, thus, noting that $\theta$ and $\theta^{\prime}$ can be expressed in terms of $\boldsymbol{d}^{\prime}, \boldsymbol{d}^{\prime \prime}, \boldsymbol{g}$, and $\boldsymbol{g}^{\prime}$, we instead choose $\boldsymbol{d}$ and $\boldsymbol{g}$ as the primary independent variables. On that basis, we next determine the conditions on $\boldsymbol{d}$ and $\boldsymbol{g}$ consistent with allowing the curvature of $\mathcal{S}$ to be piecewise continuous and recast the constraints (24) in terms of an equivalent set of constraints involving only $\boldsymbol{d}$ and $\boldsymbol{g}$. Moreover, we demonstrate that, given $\boldsymbol{d}$ and $\boldsymbol{g}$ satisfying our relaxed regularity assumptions and our alternative constraints, it is possible to construct a closed ribbon $\mathcal{S}$ and an isometric deformation $\chi$ from $\mathcal{D}$ to a ribbon $\mathcal{S}$ with piecewise continuous curvature. Due to that construction, we infer that the problem of minimizing (73) over all $\boldsymbol{d}$ and $\boldsymbol{g}$ is equivalent to the problem of minimizing (58) over all isometric deformations.

\footnotetext{
5 In its originally published form, Wunderlich's (1962) functional involves integration with respect to arclength $s$, from $s=0$ and $s=\ell$, along the midline $\mathcal{M}$ of $\mathcal{S}$. That form arises from (75) with the change of variables $\alpha \mapsto s+b$.

${ }^{6}$ See, for example, do Carmo (1976, p. 145). 


\subsection{Relaxed Regularity of the Spatial Directrix and Generatrix}

The regularity properties of $\boldsymbol{d}$ and $\boldsymbol{g}$, which stem from the assumed regularity of the deformation $\chi$, are too strong to capture all physically relevant isometric deformations of a rectangular planar strip. The need for relaxed regularity is demonstrated by Sadowsky's (1930) construction of a Möbius band with a single half-twist, which yields a surface that is $C^{1}$ but only piecewise $C^{2}$. For this reason, we hereafter relax our smoothness assumptions by assuming that

$\boldsymbol{d}$ is $C^{1}$ and $\boldsymbol{d}^{\prime}$ is piecewise $C^{1}$ and $\quad \boldsymbol{g}$ is continuous and piecewise $C^{1}$. (78)

We say that a function $\boldsymbol{u}$ defined on $[b, \ell+b]$ is piecewise $C^{1}$ if there exist a finite number of points $\alpha_{k} \in[b, \ell+b]$, with $b=\alpha_{0}<\alpha_{1}<\cdots<\alpha_{n-1}<\alpha_{n}=\ell+b$, such that $\boldsymbol{u}$ is $C^{1}$ on $\left(\alpha_{k-1}, \alpha_{k}\right)$ for $1 \leq k \leq n$ and the limits

$$
\lim _{\varepsilon \downarrow 0} \boldsymbol{u}^{\prime}\left(\alpha_{k-1}+\varepsilon\right) \quad \text { and } \quad \lim _{\varepsilon \downarrow 0} \boldsymbol{u}^{\prime}\left(\alpha_{k}-\varepsilon\right)
$$

exist for $1 \leq k \leq n$.

\subsection{Reformulation of the Constraints Ensuring that the Deformation Is an Isometry}

Our ultimate goal is to provide a framework for constructing isometric deformations that result in closed ribbons $\mathcal{S}$ that are $C^{1}$ and minimize the bending energy (58), which we have shown simplifies to (73). This dimensionally reduced expression involves the vector-valued functions $\boldsymbol{d}$ and $\boldsymbol{g}$. When working directly with (73), it is essential to ensure that those quantities generate an isometric deformation of $\mathcal{D}$, meaning that they must satisfy the constraints (24). Recalling that $c$ is given a priori by (14) or (15), $\theta$ defined by (18) is the angle between $\boldsymbol{c}^{\prime}$ and $\boldsymbol{f}$, and the parameterization (21) provides a complete covering of $\mathcal{D}$, then it transpires that the constraints (24) hold if and only if $\boldsymbol{d}$ and $\boldsymbol{g}$ satisfy the alternative collection of conditions

$$
\left|d^{\prime}\right|=1, \quad|g|=1 . \quad d^{\prime \prime} \cdot g=0, \quad \text { and } \quad\left(d^{\prime} \times g\right) \cdot g^{\prime}=0 .
$$

When using the conditions in (80) to establish the constraints (24) the function $f$ is defined by (18) with $\theta$ being the angle between $\boldsymbol{d}^{\prime}$ and $\boldsymbol{g}$. To verify the foregoing assertion, we begin by showing that (24) implies (80). It is clear that $(80)_{1,2}$ follow directly from $(24)_{1,2}$. Additionally, we notice that $(80)_{3}$ follows from (25) and that $(80)_{4}$ follows from (27) and (33). To establish the reverse implication, we begin by noticing that (80) 1,2 together with the definitions of $\boldsymbol{c}$ and $\boldsymbol{f}$ imply (24) 1,2 . Moreover, we see from (18), $(80)_{1}$, and the foregoing identification of $\theta$ with the angle between $\boldsymbol{d}^{\prime}$ and $\boldsymbol{g}$ that $(24)_{4}$ holds. Differentiating $(24)_{4}$ and utilizing $(80)_{3}$, we arrive at $(24)_{5}$. It remains only to establish $(24)_{3}$. Notice that, to ensure the reference domain $\mathcal{D}$ is completely covered, $\beta_{ \pm}$must be given by (36)-(37) if $\mathcal{S}$ is orientable or by (38)-(39) 
if $\mathcal{S}$ is nonorientable. For these expressions to be well defined, we must have

$$
\sin \theta \neq 0
$$

Constructing the Darboux frame $\{\boldsymbol{t}, \boldsymbol{m}, \boldsymbol{n}\}$ for $\boldsymbol{d}$ as in Sect. 2.4, so that, in particular, (28) holds, we find from the definition of $\theta$ and $(80)_{2}$ that $g$ has the form

$$
\boldsymbol{g}=\cos \theta \boldsymbol{t}+\sin \theta \boldsymbol{m} .
$$

Moreover, from $(28)_{1},(80)_{1,3}$, and (82), we see that

$$
0=\boldsymbol{d}^{\prime \prime} \cdot \boldsymbol{g}=\boldsymbol{t}^{\prime} \cdot \boldsymbol{g}=\left(\kappa_{g} \boldsymbol{m}+\kappa_{n} \boldsymbol{n}\right) \cdot(\cos \theta \boldsymbol{t}+\sin \theta \boldsymbol{m})=\kappa_{g} \sin \theta
$$

and, thus, from (81), that $\kappa_{g}=0$. Using this fact, (28) again, and (80) 4 , we find that

$$
\boldsymbol{g}^{\prime}=-\theta^{\prime}(\sin \theta \boldsymbol{t}-\cos \theta \boldsymbol{m})
$$

Finally, invoking (18) and (84), we obtain $(24)_{3}$.

\subsection{Construction of a Closed Ribbon with Piecewise Continuous Curvature}

We next show how to construct a closed ribbon $\mathcal{S}$ using vector-valued functions $\boldsymbol{d}$ and $g$ under the assumption that they satisfy the relaxed regularity conditions (78), the constraints (80), and the inequality (57). To describe the construction, we first fix $\boldsymbol{d}$ and $\boldsymbol{g}$ satisfying (78), (80), and (57) and define $\mathcal{S}$ by

$$
\mathcal{S}=\{\boldsymbol{d}(\alpha)+\beta \boldsymbol{g}(\alpha) \mid \alpha \in[b, \ell+b], \beta \in[-b \csc \theta(\alpha), b \csc \theta(\alpha)]\}
$$

To ensure that this defines a ribbon-like surface, it suffices to show that the parameterization defining $\mathcal{S}$ is locally injective on the interior of its domain. By the inverse function theorem, this is accomplished by showing that

$$
\begin{aligned}
0 \neq \mid\left(\boldsymbol{d}^{\prime}(\alpha)+\beta \boldsymbol{g}^{\prime}(\alpha)\right) \times & \boldsymbol{g}(\alpha)|=| \sin \theta(\alpha)-\beta \theta^{\prime} \mid, \\
& \alpha \in[b, \ell+b], \beta \in(-b \csc \theta(\alpha), b \csc \theta(\alpha)) .
\end{aligned}
$$

Using (57), in the form of the two equivalent inequalities (54) and (55), we can then show that (86) holds. The unit normal to $\mathcal{S}$ defined according to

$$
n=\frac{d^{\prime} \times g}{\left|d^{\prime} \times g\right|}
$$

is continuous by (78).

Although (78) guarantees only that $\mathcal{S}$ as defined by (85) is piecewise $C^{1}$, a consequence of the underlying structure is that $\mathcal{S}$ is everywhere $C^{1}$. To establish this claim, consider a point $\boldsymbol{p}_{\circ}=\boldsymbol{d}\left(\alpha_{\circ}\right)+\beta_{\circ} \boldsymbol{g}\left(\alpha_{\circ}\right)$ on $\mathcal{S}$. Notice that if $\boldsymbol{g}$ is $C^{1}$ at $\alpha_{\circ}$, then $\mathcal{S}$ is 
$C^{1}$ at $\boldsymbol{p}_{\circ}$. Thus, we need only consider situations in which $\boldsymbol{g}^{\prime}$ is discontinuous at $\alpha_{\circ}$. Let $\mathcal{S}^{ \pm}$be sets defined such that

$$
\left.\begin{array}{l}
\mathcal{S}^{+}=\left\{\boldsymbol{d}(\alpha)+\beta \boldsymbol{g}(\alpha) \mid \alpha_{\circ} \leq \alpha \leq \ell+b, \beta \in[-b \csc \theta(\alpha), b \csc \theta(\alpha)]\right\}, \\
\mathcal{S}^{-}=\left\{\boldsymbol{d}(\alpha)+\beta \boldsymbol{g}(\alpha) \mid b \leq \alpha \leq \alpha_{\circ}, \beta \in[-b \csc \theta(\alpha), b \csc \theta(\alpha)]\right\} .
\end{array}\right\}
$$

Notice that $\mathcal{S}^{ \pm}$is, at $\boldsymbol{p}_{\circ}$, locally diffeomorphic to a half-disc. To specify the underlying diffeomorphisms, consider the plane

$$
\mathcal{A}=\left\{\boldsymbol{u} \in \mathcal{E} \mid\left(\boldsymbol{u}-\boldsymbol{p}_{\circ}\right) \cdot \boldsymbol{n}\left(\boldsymbol{p}_{\circ}\right)=0\right\} .
$$

Since $\mathcal{S}^{+}$and $\mathcal{S}^{-}$share the same normal at $\boldsymbol{p}_{\circ}, \mathcal{A}$ is tangent to both of these surfaces at $\boldsymbol{p}_{\circ}$. For any $\varepsilon>0$, let $B_{\varepsilon}$ denote the closed ball of radius $\varepsilon$ in $\mathcal{A}$ centered at $\boldsymbol{p}_{\circ}$. The ruling $\mathcal{L}_{\circ}$ that passes through $\boldsymbol{p}_{\circ}$ lies on $\mathcal{A}$ and, hence, divides $B_{\varepsilon}$ into two halves, say $B_{\varepsilon}^{+}$and $B_{\varepsilon}^{-}$, that correspond to whichever of $\mathcal{S}^{+}$or $\mathcal{S}^{-}$is closest. There is an $\varepsilon>0$ and $C^{1}$ functions $\varphi^{ \pm}: B_{\varepsilon}^{ \pm} \rightarrow \mathcal{S}^{ \pm} \subset \mathcal{E}$, such that $(i) \varphi^{ \pm}\left(\boldsymbol{p}_{\circ}\right)=\boldsymbol{p}_{\circ}$ and $(i i) \varphi^{ \pm}(\boldsymbol{u})-\boldsymbol{u}$ is parallel to $\boldsymbol{n}\left(\boldsymbol{p}_{\circ}\right)$ for all $\boldsymbol{u} \in B_{\varepsilon}^{ \pm}$. Here, $\boldsymbol{\varphi}^{ \pm}$represents $\mathcal{S}^{ \pm}$near $\boldsymbol{p}_{\circ}$ using the tangent space $\mathcal{A}$ of $\mathcal{S}^{ \pm}$at $\boldsymbol{p}_{\circ}$. An analytical consequence of the geometric requirement that $\mathcal{A}$ be tangent to $\mathcal{S}^{ \pm}$along $\mathcal{L}_{\circ}$ is that $\varphi^{ \pm}$and the natural injection of $\mathcal{A}$ into $\mathcal{E}$ have the same gradient along $\mathcal{L}_{\circ}$. Thus,

$$
\left(\nabla \varphi^{ \pm}(p)\right) v=v, \quad p \in B_{\varepsilon}^{ \pm} \cap \mathcal{L}_{\circ}, \quad \boldsymbol{v} \in\{\boldsymbol{n}\}^{\perp} .
$$

Next, defining $\varphi: B_{\varepsilon} \rightarrow \mathcal{S}$ by

$$
\varphi(u)= \begin{cases}\varphi^{+}(u), & u \in B_{\varepsilon}^{+} \backslash \mathcal{L}_{\circ}, \\ u, & u \in B_{\varepsilon} \cap \mathcal{L}_{\circ}, \\ \varphi^{-}(u), & u \in B_{\varepsilon}^{-} \backslash \mathcal{L}_{\circ}\end{cases}
$$

we see that, due to the regularity of $\varphi^{ \pm}$and (90), $\varphi$ is $C^{1}$. Thus, we conclude that $\mathcal{S}$ is $C^{1}$ at $\boldsymbol{p}_{\circ}$ as claimed.

\subsection{Construction of an Isometric Deformation from a Rectangular Strip to a Closed Ribbon with Piecewise Continuous Curvature}

Having shown how a closed ribbon $\mathcal{S}$ with piecewise continuous curvature can be constructed, we next show how the underlying isometric deformation from the rectangular strip $\mathcal{D}$ to $\mathcal{S}$ can be constructed. In so doing, we must distinguish whether $\mathcal{S}$ is orientable or nonorientable, as determined by which alternate form, (14) or (15), of the referential directrix $\boldsymbol{c}$ is used. Granted that $\boldsymbol{d}$ and $\boldsymbol{g}$ satisfy the relaxed regularity conditions (78) and the constraints (57) and (80), we may define the parameterization $\hat{\boldsymbol{r}}$ on $[b, \ell+b] \times \mathbb{R}$. The bijection $\hat{\alpha}$ introduced in Sect. 2.5 can then be used to extend the domain of $\hat{\boldsymbol{r}}$ from $\mathcal{P}$ to $[0, \ell+2 b] \times \mathbb{R}$ and consequently ensure that (43)-(44) hold if $\mathcal{S}$ is orientable or that (51) holds if $\mathcal{S}$ is nonorientable. This requires extending 
$\boldsymbol{d}$ to the interval $[0, \ell+2 b]$. Recalling that $\boldsymbol{c}$ is given by either (14) or (15) and that $\boldsymbol{f}$ is defined by (18), with $\theta$ being the angle between $\boldsymbol{d}^{\prime}$ and $\boldsymbol{g}$, we can define the parameterization (21). The constraints (24) are satisfied since (80) hold. Thus, they induce an isometric deformation $\chi$ from $\mathcal{D}$ to $\mathcal{S}$. We can use the inequality (57) to show that the parameterization $\hat{\boldsymbol{x}}$ of $\mathcal{D}$ is locally injective, just as we previously did for the parametrization $\hat{\boldsymbol{r}}$ of $\mathcal{S}$ and, hence, we may conclude that the deformation $\chi$ is also locally injective. Since $\mathcal{D}$ and $\mathcal{S}$ are both $C^{1}$, a result of Myers and Steenrod (1939) ensures that $\chi$ is $C^{1}$.

It is important to note here that the deformation $\chi$ constructed as described need not be injective, but only locally injective. In the mathematics literature, $\chi$ would be called an isometric immersion. The properties of such immersions have been studied by Pakzad (2004), Müller and Pakzad (2005), and Hornung (2011a,c), among others. To ensure that the constructed $\chi$ is injective, it would be necessary to require that $\boldsymbol{d}$ and $\boldsymbol{g}$ satisfy an appropriate global constraint, the formulation and analysis of which we leave to a future work. In a related work, Halpern and Weaver (1977) discussed the restrictions on $b / \ell$ that guarantee the existence of an isometric immersion from a rectangular strip of length $\ell$ and width $2 b$ to a half-twist Möbius band along with the additional requirements on $b / \ell$ that guarantee the existence of an isometric embedding - that is, an injective isometric emersion.

\section{Necessary Conditions for Equilibrium}

We next specify the variational problem associated with finding an equilibrium shape $\mathcal{S}$ of the rectangular strip $\mathcal{D}$ that can undergo only isometric deformations induced by one of the connecting edge conditions (10) or (11) while leaving $\partial \mathcal{S}$ free of traction and couple traction. To begin, we recall from the previous section that, to construct $\mathcal{S}$, it suffices to find vector-valued functions $\boldsymbol{d}$ and $\boldsymbol{g}$ defined on $[b, \ell+b]$ and consistent with the constraints (57) and (80) that minimize the dimensionally reduced bending energy (73).

To incorporate the constraints (57) and (80), we consider the augmented bending energy functional

$$
\begin{aligned}
E^{*}[\boldsymbol{d}, \boldsymbol{g}]= & \int_{b}^{\ell+b}\left(F\left(\boldsymbol{d}^{\prime}, \boldsymbol{g}, \boldsymbol{g}^{\prime}\right)\left|\boldsymbol{d}^{\prime \prime}\right|^{2}-\lambda\left(\left|\boldsymbol{d}^{\prime}\right|^{2}-1\right)\right. \\
& \left.-\chi\left(|\boldsymbol{g}|^{2}-1\right)-\gamma \boldsymbol{d}^{\prime \prime} \cdot \boldsymbol{g}-\nu\left(\boldsymbol{d}^{\prime} \times \boldsymbol{g}\right) \cdot \boldsymbol{g}^{\prime}-\omega G\left(\boldsymbol{d}^{\prime}, \boldsymbol{g}, \boldsymbol{g}^{\prime}\right)\right) \mathrm{d} \alpha,
\end{aligned}
$$

where $F$ and $G$ are given by

$$
\begin{aligned}
F\left(\boldsymbol{d}^{\prime}, \boldsymbol{g}, \boldsymbol{g}^{\prime}\right) & =\frac{\mu}{2 \theta^{\prime} \sin ^{2} \theta} \log \frac{\sin ^{2} \theta+b \theta^{\prime}}{\sin ^{2} \theta-b \theta^{\prime}}, \\
& =\frac{\mu}{2\left(\boldsymbol{d}^{\prime} \cdot \boldsymbol{g}^{\prime}\right) \sqrt{1-\left(\boldsymbol{d}^{\prime} \cdot \boldsymbol{g}\right)^{2}}} \log \frac{\left(1-\left(\boldsymbol{d}^{\prime} \cdot \boldsymbol{g}\right)^{2}\right)^{3 / 2}+b\left(\boldsymbol{d}^{\prime} \cdot \boldsymbol{g}^{\prime}\right)}{\left(1-\left(\boldsymbol{d}^{\prime} \cdot \boldsymbol{g}\right)^{2}\right)^{3 / 2}-b\left(\boldsymbol{d}^{\prime} \cdot \boldsymbol{g}^{\prime}\right)}
\end{aligned}
$$


and

$$
G\left(\boldsymbol{d}^{\prime}, \boldsymbol{g}, \boldsymbol{g}^{\prime}\right)=\sin ^{4} \theta-b^{2} \theta^{2}=\left(1-\left(\boldsymbol{d}^{\prime} \cdot \boldsymbol{g}\right)^{2}\right)^{2}-\frac{b^{2}\left(\boldsymbol{d}^{\prime} \cdot \boldsymbol{g}^{\prime}\right)^{2}}{1-\left(\boldsymbol{d}^{\prime} \cdot \boldsymbol{g}\right)^{2}}
$$

Here $\lambda, \chi, \gamma$, and $v$ are arclength dependent Lagrange multipliers and $\omega$ is an arclength dependent Karush-Kuhn-Tucker multiplier.

The connecting edge conditions (10) and (11) must be formulated in terms of $\boldsymbol{d}$ and $g$, and we do this in the next two subsections, considering the orientable and nonorientable cases separately. In addition, the extent to which the ribbon $\mathcal{S}$ is twisted must also be specified. The edge condition (10) can be met in the absence of twist, in which case the equilibrium shape of $\mathcal{S}$ would be cylindrical and, by the regularity assumptions (78), smooth. It is also possible, however, to satisfy (10) in the presence of any number of full twists, assuming, as stated previously, that the half-width-to-length aspect ratio $b / \ell$ is sufficiently small. An analogous statement involving half-twists applies to (11). To quantify the number of twists present in either case, we will use the linking number between the midline $\mathcal{M}$ of $\mathcal{S}$ and a perturbation of $\mathcal{M}$ along $\mathcal{S}$. Although knotted strips are also compatible with the connecting edge conditions (10) and (11), we disregard that possibility in the present work.

\subsection{Orientable Closed Ribbons}

If $\mathcal{D}$ is isometrically deformed by holding one of its short ends fixed and subjecting the other short end to any number $k$ full twists before satisfying (10), then the resulting orientable surface $\mathcal{S}$ is not a closed ribbon unless $\boldsymbol{d}$ and $\boldsymbol{g}$ satisfy

$$
\boldsymbol{d}(b)=\boldsymbol{d}(\ell+b), \quad \boldsymbol{d}^{\prime}(b)=\boldsymbol{d}^{\prime}(\ell+b), \quad \text { and } \quad \boldsymbol{g}(b)=\boldsymbol{g}(\ell+b) .
$$

Consider a perturbation $\mathcal{M}_{\varepsilon}$ of the midline $\mathcal{M}$ of $\mathcal{S}$, in the direction of $\boldsymbol{g}$, with parameterization

$$
\boldsymbol{d}_{\varepsilon}(\alpha)=\boldsymbol{d}(\alpha)+\varepsilon \boldsymbol{g}(\alpha), \quad \alpha \in[b, \ell+b],
$$

where, to ensure that $\boldsymbol{d}_{\varepsilon}$ is on the surface generated by $\boldsymbol{d}$ and $\boldsymbol{g}, \varepsilon>0$ satisfies

$$
\varepsilon<b \csc \theta_{\circ}, \quad \text { with } \quad \theta_{\circ}=\min _{b \leq \alpha \leq \ell+b} \theta(\alpha) .
$$

Then, the linking number $\operatorname{Lk}\left(\mathcal{M}, \mathcal{M}_{\varepsilon}\right)$ between the midline $\mathcal{M}$ parameterized by $\boldsymbol{d}$ and its perturbation $\mathcal{M}_{\varepsilon}$ parameterized by $\boldsymbol{d}_{\varepsilon}$ must obey

$$
\operatorname{Lk}\left(\mathcal{M}, \mathcal{M}_{\varepsilon}\right)=\int_{b}^{\ell+b} \int_{b}^{\ell+b} \frac{\left(\boldsymbol{d}(\alpha)-\boldsymbol{d}_{\varepsilon}(\tilde{\alpha})\right) \cdot\left(\boldsymbol{d}^{\prime}(\alpha) \times \boldsymbol{d}_{\varepsilon}^{\prime}(\tilde{\alpha})\right)}{4 \pi\left|\boldsymbol{d}(\alpha)-\boldsymbol{d}_{\varepsilon}(\tilde{\alpha})\right|^{3}} \mathrm{~d} \alpha \mathrm{d} \tilde{\alpha}=k .
$$

The value of $\operatorname{Lk}\left(\mathcal{M}, \mathcal{M}_{\varepsilon}\right)$ determined by (98) does not depend on the choice of $\varepsilon$. Since there are two directions in which the twist can be performed, $k$ may be positive or negative. In the absence of twist, $k=0$. 


\subsection{Nonorientable Closed Ribbons}

If $\mathcal{D}$ is isometrically deformed by holding one of its short ends fixed and subjecting the other short end to any odd number $k \neq 0$ half-twists before imposing (11), then the resulting nonorientable surface $\mathcal{S}$ is not a closed ribbon unless $\boldsymbol{d}$ and $\boldsymbol{g}$ satisfy

$$
\boldsymbol{d}(b)=\boldsymbol{d}(\ell+b), \quad \boldsymbol{d}^{\prime}(b)=\boldsymbol{d}^{\prime}(\ell+b), \quad \text { and } \quad \boldsymbol{g}(b)=-\boldsymbol{g}(\ell+b) .
$$

Proceeding much as in the orientable case, consider a perturbation $\mathcal{M}_{\varepsilon}$ of the midline $\mathcal{M}$ of $\mathcal{S}$, in the direction of $\boldsymbol{g}$, with parameterization

$$
\boldsymbol{d}_{\varepsilon}(s)= \begin{cases}\boldsymbol{d}(\alpha)+\varepsilon \boldsymbol{g}(\alpha), & \alpha \in[b, \ell+b), \\ \boldsymbol{d}(\alpha-\ell)-\varepsilon \boldsymbol{g}(\alpha-\ell), & \alpha \in[\ell+b, 2 \ell+b],\end{cases}
$$

where $\varepsilon>0$ satisfies (97) and the interval $[b, 2 \ell+b]$ for the arclength along $\mathcal{M}_{\varepsilon}$ is required to ensure that it is closed. Then, the linking number $\operatorname{Lk}\left(\mathcal{M}, \mathcal{M}_{\varepsilon}\right)$ between the midline $\mathcal{M}$ parameterized by $\boldsymbol{d}$ and its perturbation $\mathcal{M}_{\varepsilon}$ parameterized by $\boldsymbol{d}_{\varepsilon}$ must obey

$$
\operatorname{Lk}\left(\mathcal{M}, \mathcal{M}_{\varepsilon}\right)=\int_{b}^{2 \ell+b} \int_{b}^{\ell+b} \frac{\left(\boldsymbol{d}(\alpha)-\boldsymbol{d}_{\varepsilon}(\tilde{\alpha})\right) \cdot\left(\boldsymbol{d}^{\prime}(\alpha) \times \boldsymbol{d}_{\varepsilon}^{\prime}(\tilde{\alpha})\right)}{4 \pi\left|\boldsymbol{d}(\alpha)-\boldsymbol{d}_{\varepsilon}(\tilde{\alpha})\right|^{3}} \mathrm{~d} \alpha \mathrm{d} \tilde{\alpha}=k
$$

Notice that (101) differs from (98) not only in the interpretation of $k$ as the number of half-twists (in contrast to whole-twists) but also in the upper limit of the integral over $\mathcal{M}_{\varepsilon}$. As in the orientable case, the value of $\operatorname{Lk}\left(\mathcal{M}, \mathcal{M}_{\varepsilon}\right)$ determined by (101) does not depend on the choice of $\varepsilon$ and $k$ is signed depending on the direction of twisting.

\subsection{Euler-Lagrange Equations}

Here, we derive the first-order equilibrium conditions necessary for $\boldsymbol{d}$ and $\boldsymbol{g}$ to be a minimizer of the augmented bending energy (92). Recalling that the functions $\boldsymbol{d}$ and $\boldsymbol{g}$ satisfy (78), and letting $\delta \boldsymbol{d}$ and $\delta \boldsymbol{g}$ denote smooth variations of $\boldsymbol{d}$ and $\boldsymbol{g}$ that are consistent with one of the two alternative collections, (95) or (99), of edge conditions, we find that the first variation $\delta E^{*}[\boldsymbol{d}, \boldsymbol{g}](\delta \boldsymbol{d}, \delta \boldsymbol{g})$ of $E^{*}[\boldsymbol{d}, \boldsymbol{g}]$ takes the form

$$
\begin{aligned}
\delta E^{*}[\boldsymbol{d}, \boldsymbol{g}](\delta \boldsymbol{d}, \delta \boldsymbol{g})= & \int_{b}^{\ell+b}\left(2 F \boldsymbol{d}^{\prime \prime} \cdot \delta \boldsymbol{d}^{\prime \prime}+\left|\boldsymbol{d}^{\prime \prime}\right|^{2}\left(F_{\boldsymbol{d}^{\prime}} \cdot \delta \boldsymbol{d}^{\prime}+F_{\boldsymbol{g}} \cdot \delta \boldsymbol{g}+F_{\boldsymbol{g}^{\prime}} \cdot \delta \boldsymbol{g}^{\prime}\right)\right. \\
& -2 \lambda \boldsymbol{d}^{\prime} \cdot \delta \boldsymbol{d}^{\prime}-2 \chi \boldsymbol{g} \cdot \delta \boldsymbol{g}-\gamma\left(\boldsymbol{d}^{\prime \prime} \cdot \delta \boldsymbol{g}+\boldsymbol{g} \cdot \delta \boldsymbol{d}^{\prime \prime}\right) \\
& -v\left(\left(\boldsymbol{g} \times \boldsymbol{g}^{\prime}\right) \cdot \delta \boldsymbol{d}^{\prime}-\left(\boldsymbol{d}^{\prime} \times \boldsymbol{g}^{\prime}\right) \cdot \delta \boldsymbol{g}+\left(\boldsymbol{d}^{\prime} \times \boldsymbol{g}\right) \cdot \delta \boldsymbol{g}^{\prime}\right) \\
& \left.-\omega\left(G_{\boldsymbol{d}^{\prime}} \cdot \delta \boldsymbol{d}^{\prime}+G_{\boldsymbol{g}} \cdot \delta \boldsymbol{g}+G_{\boldsymbol{g}^{\prime}} \cdot \delta \boldsymbol{g}^{\prime}\right)\right) \mathrm{d} \alpha
\end{aligned}
$$


where on defining

$$
\left.\begin{array}{l}
M(\xi, \zeta)=\frac{3 b \xi \mu}{b^{2} \zeta^{2}-\xi^{6}}-\frac{\mu}{2 \xi^{2} \zeta} \log \frac{\xi^{3}+b \zeta}{\xi^{3}-b \zeta}, \\
N(\xi, \zeta)=\frac{\mu b \xi^{2}}{\left(\xi^{6}-b^{2} \zeta^{2}\right) \zeta}-\frac{\mu}{2 \xi \zeta^{2}} \log \frac{\xi^{3}+b \zeta}{\xi^{3}-b \zeta}, \\
P(\xi, \zeta)=3 \xi^{3}+2 b^{2} \zeta^{2} / \xi^{3}, \\
Q(\xi, \zeta)=\xi^{4}-2 b^{2} \zeta / \xi^{2},
\end{array}\right\}
$$

the various derivatives of $F$ and $G$ are given by

$$
\left.\begin{array}{l}
F_{d^{\prime}}\left(d^{\prime}, g, g^{\prime}\right)=\frac{M\left(\sqrt{1-\left(d^{\prime} \cdot g\right)^{2}}, d^{\prime} \cdot g^{\prime}\right)}{\sqrt{1-\left(d^{\prime} \cdot g\right)^{2}}}\left(d^{\prime} \cdot g\right) g+N\left(\sqrt{1-\left(d^{\prime} \cdot g\right)^{2}}, d^{\prime} \cdot g^{\prime}\right) g^{\prime} \\
F_{g}\left(d^{\prime}, g, g^{\prime}\right)=\frac{M\left(\sqrt{1-\left(d^{\prime} \cdot g\right)^{2}}, d^{\prime} \cdot g^{\prime}\right)}{\sqrt{1-\left(d^{\prime} \cdot g\right)^{2}}}\left(d^{\prime} \cdot g\right) d^{\prime} \\
F_{g^{\prime}}\left(d^{\prime}, g, g^{\prime}\right)=N\left(\sqrt{1-\left(d^{\prime} \cdot g\right)^{2}}, d^{\prime} \cdot g^{\prime}\right) d^{\prime}
\end{array}\right\}
$$

and

$$
\begin{aligned}
& G_{d^{\prime}}\left(d^{\prime}, g, g^{\prime}\right)=\frac{P\left(\sqrt{1-\left(d^{\prime} \cdot g\right)^{2}}, d^{\prime} \cdot g^{\prime}\right)}{\sqrt{1-\left(d^{\prime} \cdot g\right)^{2}}}\left(d^{\prime} \cdot g\right) g+Q\left(\sqrt{1-\left(d^{\prime} \cdot g\right)^{2}}, d^{\prime} \cdot g^{\prime}\right) g^{\prime}, \\
& G_{g}\left(d^{\prime}, g, g^{\prime}\right)=\frac{P\left(\sqrt{1-\left(d^{\prime} \cdot g\right)^{2}}, d^{\prime} \cdot g^{\prime}\right)}{\sqrt{1-\left(d^{\prime} \cdot g\right)^{2}}}\left(d^{\prime} \cdot g\right) d^{\prime}, \\
& G_{g^{\prime}}\left(d^{\prime}, g, g^{\prime}\right)=Q\left(\sqrt{1-\left(d^{\prime} \cdot g\right)^{2}}, d^{\prime} \cdot g^{\prime}\right) d^{\prime} .
\end{aligned}
$$

Setting (102) equal to zero gives a weak statement of the conditions that must hold in equilibrium.

Granted that $\boldsymbol{d}$ is piecewise $C^{3}$ and $\boldsymbol{g}$ is piecewise $C^{2}$, we find upon integrating by parts several times that (102) can be expressed as

$$
\begin{aligned}
\delta E^{*}[\boldsymbol{d}, \boldsymbol{g}](\delta \boldsymbol{d}, \delta \boldsymbol{g})= & \int_{b}^{\ell+b}(\boldsymbol{\varphi} \cdot \delta \boldsymbol{d}+\boldsymbol{\psi} \cdot \delta \boldsymbol{g}) \mathrm{d} \alpha+\left.\left(\boldsymbol{\xi} \cdot \delta \boldsymbol{d}+\boldsymbol{\eta} \cdot \delta \boldsymbol{d}^{\prime}+\boldsymbol{\zeta} \cdot \boldsymbol{g}\right)\right|_{b} ^{\ell+b} \\
& +\left.\lim _{\varepsilon \downarrow 0} \sum_{k=2}^{n-1}\left(\boldsymbol{\xi} \cdot \delta \boldsymbol{d}+\boldsymbol{\eta} \cdot \delta \boldsymbol{d}^{\prime}+\boldsymbol{\zeta} \cdot \boldsymbol{g}\right)\right|_{\alpha_{k}-\varepsilon} ^{\alpha_{k}+\varepsilon},
\end{aligned}
$$


where $\varphi, \psi, \xi, \eta$, and $\zeta$ are given by

$$
\begin{aligned}
& \varphi=\left(2 F d^{\prime \prime}\right)^{\prime \prime}-\left(\left|d^{\prime \prime}\right|^{2} F_{d^{\prime}}\right)^{\prime}+\left(2 \lambda d^{\prime}\right)^{\prime} \\
& -(\gamma \boldsymbol{g})^{\prime \prime}+\left(\omega \boldsymbol{g} \times \boldsymbol{g}^{\prime}\right)^{\prime}+\left(\omega G_{\boldsymbol{d}^{\prime}}\right)^{\prime}, \\
& \boldsymbol{\psi}=\left|\boldsymbol{d}^{\prime \prime}\right|^{2} F_{\boldsymbol{g}}-\left(\left|\boldsymbol{d}^{\prime \prime}\right|^{2} F_{\boldsymbol{g}^{\prime}}\right)^{\prime}-2 \chi \boldsymbol{g}-\gamma \boldsymbol{d}^{\prime \prime} \\
& +\left(v \boldsymbol{d}^{\prime} \times \boldsymbol{g}\right)^{\prime}+v \boldsymbol{d}^{\prime} \times \boldsymbol{g}^{\prime}-\omega G_{\boldsymbol{g}}+\left(\omega G_{\boldsymbol{g}^{\prime}}\right)^{\prime}, \\
& \boldsymbol{\xi}=\left|\boldsymbol{d}^{\prime \prime}\right|^{2} F_{\boldsymbol{d}^{\prime}}-\left(2 F \boldsymbol{d}^{\prime \prime}\right)^{\prime}-2 \lambda \boldsymbol{d}^{\prime}+(\gamma \boldsymbol{g})^{\prime}-v \boldsymbol{g} \times \boldsymbol{g}^{\prime}-\omega G_{\boldsymbol{d}^{\prime}}, \\
& \eta=2 F d^{\prime \prime}-\gamma g \\
& \zeta=\left|\boldsymbol{d}^{\prime \prime}\right|^{2} F_{\boldsymbol{g}^{\prime}}-v \boldsymbol{d}^{\prime} \times \boldsymbol{g}-\omega G_{\boldsymbol{g}^{\prime}} .
\end{aligned}
$$

From the requirement that the first variation $\delta E^{*}[\boldsymbol{d}, \boldsymbol{g}](\delta \boldsymbol{d}, \delta \boldsymbol{g})$ of $E^{*}[\boldsymbol{d}, \boldsymbol{g}]$ must vanish for all admissible variations $\delta \boldsymbol{d}$ and $\delta \boldsymbol{g}$, we obtain Euler-Lagrange equations in the form of ordinary differential equations

$$
\left.\begin{array}{r}
\left(\left(2 F \boldsymbol{d}^{\prime \prime}\right)^{\prime}-\left|\boldsymbol{d}^{\prime \prime}\right|^{2} F_{\boldsymbol{d}^{\prime}}+2 \lambda \boldsymbol{d}^{\prime}-(\gamma \boldsymbol{g})^{\prime}+v \boldsymbol{g} \times \boldsymbol{g}^{\prime}+\omega G_{\boldsymbol{d}^{\prime}}\right)^{\prime}=\mathbf{0}, \\
\left(\left|\boldsymbol{d}^{\prime \prime}\right|^{2} F_{\boldsymbol{g}^{\prime}}\right)^{\prime}-\left|\boldsymbol{d}^{\prime \prime}\right|^{2} F_{\boldsymbol{g}}+2 \chi \boldsymbol{g}-\gamma \boldsymbol{d}^{\prime \prime}-\left(\nu \boldsymbol{d}^{\prime} \times \boldsymbol{g}\right)^{\prime} \\
-v \boldsymbol{d}^{\prime} \times \boldsymbol{g}^{\prime}+\omega G_{\boldsymbol{g}}-\left(\omega G_{\boldsymbol{g}^{\prime}}\right)^{\prime}=\mathbf{0},
\end{array}\right\}
$$

which apply on the interval $(b, l+b)$, matching conditions

$$
\left.\begin{array}{c}
\left(\left(2 F \boldsymbol{d}^{\prime \prime}\right)^{\prime}-\left|\boldsymbol{d}^{\prime \prime}\right|^{2} F_{\boldsymbol{d}^{\prime}}+2 \lambda \boldsymbol{d}^{\prime}-(\gamma \boldsymbol{g})^{\prime}+v \boldsymbol{g} \times \boldsymbol{g}^{\prime}+\omega G_{\boldsymbol{d}^{\prime}}\right)(\ell+b) \\
=\left(\left(2 F \boldsymbol{d}^{\prime \prime}\right)^{\prime}-\left|\boldsymbol{d}^{\prime \prime}\right|^{2} F_{\boldsymbol{d}^{\prime}}+2 \lambda \boldsymbol{d}^{\prime}-(\gamma \boldsymbol{g})^{\prime}+\nu \boldsymbol{g} \times \boldsymbol{g}^{\prime}+\omega G_{\boldsymbol{d}^{\prime}}\right)(b), \\
\left(2 F \boldsymbol{d}^{\prime \prime}-\gamma \boldsymbol{g}\right)(\ell+b)=\left(2 F \boldsymbol{d}^{\prime \prime}-\gamma \boldsymbol{g}\right)(b), \\
\left(\left|\boldsymbol{d}^{\prime \prime}\right|^{2} F_{\boldsymbol{g}^{\prime}}-v \boldsymbol{d}^{\prime} \times \boldsymbol{g}-\omega G_{\boldsymbol{g}^{\prime}}\right)(\ell+b)= \pm\left(\left|\boldsymbol{d}^{\prime \prime}\right|^{2} F_{\boldsymbol{g}^{\prime}}-v \boldsymbol{d}^{\prime} \times \boldsymbol{g}-\omega G_{\boldsymbol{g}^{\prime}}\right)(b),
\end{array}\right\}
$$

and jump conditions

$$
\left.\begin{array}{r}
\llbracket\left(2 F \boldsymbol{d}^{\prime \prime}\right)^{\prime}-\left|\boldsymbol{d}^{\prime \prime}\right|^{2} F_{\boldsymbol{d}^{\prime}}+2 \lambda \boldsymbol{d}^{\prime}-(\gamma \boldsymbol{g})^{\prime}+v \boldsymbol{g} \times \boldsymbol{g}^{\prime}+\omega G_{\boldsymbol{d}^{\prime}} \rrbracket=\mathbf{0}, \\
\llbracket 2 F \boldsymbol{d}^{\prime \prime}-\gamma \boldsymbol{g} \rrbracket=\mathbf{0}, \\
\llbracket\left|\boldsymbol{d}^{\prime \prime}\right|^{2} F_{\boldsymbol{g}^{\prime}}-v \boldsymbol{d}^{\prime} \times \boldsymbol{g}-\omega G_{\boldsymbol{g}^{\prime}} \rrbracket=\mathbf{0} .
\end{array}\right\}
$$

Notice the sign on the right-hand side of the matching condition $(109)_{3}$ is dictated by the orientability of $\mathcal{S}$ - the plus sign applies if $\mathcal{S}$ is orientable and the minus sign applies otherwise. 
In addition to the equilibrium conditions (108)-(110) and the constraints (80), it is also necessary to satisfy the conditions

$$
0=\omega\left(\sin ^{4} \theta-b^{2} \theta^{\prime 2}\right) \quad \text { and } \quad \omega \geq 0
$$

associated with the Karush-Kuhn-Tucker multiplier, together with either the consequence (95) of the edge condition (10) and the specification (98) of the linking number $k \in \mathbb{Z}$, which apply if $\mathcal{S}$ is orientable, or the consequence (99) of the edge condition (11) and the specification (101) of the linking number $k$ satisfying $k \in \mathbb{Z} \backslash\{0\}$, which apply if $\mathcal{S}$ is nonorientable.

It is worth emphasizing that the equilibrium equations we obtain are written directly in terms of two vector-valued functions: the directrix $\boldsymbol{d}$ and the generatrix $\boldsymbol{g}$, the two quantities that are necessary and sufficient to construct a surface $\mathcal{S}$ and a locally injective isometric deformation from $\mathcal{D}$ to $\mathcal{S}$. This is in contrast to other equilibrium equations found in the literature. Whereas Starostin and van der Heijden (2015) write their equilibrium equations in terms of the Frenet-Serret frame of the midline and its derivatives, Dias and Audoly (2015) write their equilibrium equations using the curvature and torsion of the midline. Hornung's (2011b) equations are very different in structure since they are not traditional boundary value problems, but rather are in a form amenable to establishing regularity results.

\subsection{Comments on the Matching Conditions}

We next interpret the matching conditions (109).

- (109) $)_{1}$ : This condition expresses the requirement that the forces at the points $\boldsymbol{d}(b)$ and $\boldsymbol{d}(\ell+b)$ of $\mathcal{S}$ be equal. Moreover, these forces act to ensure that $\boldsymbol{d}(b)=$ $\boldsymbol{d}(\ell+b)$, and hence, that the midline $\mathcal{M}$ is closed.

- $(109)_{2}$ : Bearing in mind that $\boldsymbol{d}^{\prime \prime}$ and $\boldsymbol{g}$ are orthogonal as a consequence of $(80)_{3}$, we first observe that $(109)_{2}$ is equivalent to

$$
(\gamma \boldsymbol{g})(b)=(\gamma \boldsymbol{g})(\ell+b) \quad \text { and } \quad\left(F \boldsymbol{d}^{\prime \prime}\right)(b)=\left(F \boldsymbol{d}^{\prime \prime}\right)(\ell+b) \text {. }
$$

If the condition $(112)_{1}$ is applied in conjunction with either $(95)_{3}$ or $(99)_{3}$, it follows that the Lagrange multiplier $\gamma$ satisfies either $(95)_{3}$ or $(99)_{3}$, respectively, with $g$ replaced by $\gamma$. Condition $(112)_{2}$ expresses the requirement that the bending moments

$$
\left(F\left(\boldsymbol{d}^{\prime}, \boldsymbol{g}, \boldsymbol{g}^{\prime}\right) \boldsymbol{d}^{\prime \prime}\right)(b) \quad \text { and } \quad\left(F\left(\boldsymbol{d}^{\prime}, \boldsymbol{g}, \boldsymbol{g}^{\prime}\right) \boldsymbol{d}^{\prime \prime}\right)(\ell+b)
$$

at the points $\boldsymbol{d}(b)$ and $\boldsymbol{d}(\ell+b)$ are equal. Moreover, these bending moments act to ensure that $\boldsymbol{d}^{\prime}(b)=\boldsymbol{d}^{\prime}(\ell+b)$, and hence, that the tangent to $\mathcal{M}$ is smooth at the point of closure.

- (109) 3 : Upon noticing that $F_{\boldsymbol{g}^{\prime}}\left(\boldsymbol{d}^{\prime}, \boldsymbol{g}, \boldsymbol{g}^{\prime}\right)$ and $G_{\boldsymbol{g}^{\prime}}\left(\boldsymbol{d}^{\prime}, \boldsymbol{g}, \boldsymbol{g}^{\prime}\right)$ are tangent to $\mathcal{M}$, we see that $(109)_{3}$ expresses the requirement that the twisting moments

$$
\left(\left|\boldsymbol{d}^{\prime \prime}\right|^{2} F_{\boldsymbol{g}^{\prime}}\left(\boldsymbol{d}^{\prime}, \boldsymbol{g}, \boldsymbol{g}^{\prime}\right)-v \boldsymbol{d}^{\prime} \times \boldsymbol{g}-\omega G_{\boldsymbol{g}^{\prime}}\right)(b)
$$


and

$$
\left(\left|\boldsymbol{d}^{\prime \prime}\right|^{2} F_{\boldsymbol{g}^{\prime}}\left(\boldsymbol{d}^{\prime}, \boldsymbol{g}, \boldsymbol{g}^{\prime}\right)-v \boldsymbol{d}^{\prime} \times \boldsymbol{g}-\omega G_{\boldsymbol{g}^{\prime}}(\ell+b)\right.
$$

at the points $\boldsymbol{d}(b)$ and $\boldsymbol{d}(\ell+b)$ of $\mathcal{S}$ are of equal magnitude and orientation consistent with the orientability of $\mathcal{S}$. Moreover, these twisting moments act to ensure that $\boldsymbol{g}(b)=\boldsymbol{g}(\ell+b)$, and hence, that the short edges of $\mathcal{D}$ are continuously joined.

\subsection{Nonuniqueness of the Rulings on Flat Portions of the Ribbon}

We next show that on intervals where $\boldsymbol{d}^{\prime \prime}=\mathbf{0}$, namely the intervals associated with the flat subregions of $\mathcal{S}$, no solution of (108) and (110) is unique. To see this, we consider a maximal interval $\left[\alpha_{1}, \alpha_{2}\right]$ upon which $\boldsymbol{d}^{\prime \prime}=\mathbf{0}$. Since the vector $\boldsymbol{t}=\boldsymbol{d}^{\prime}$ is constant on $\left[\alpha_{1}, \alpha_{2}\right]$, we find that the Euler-Lagrange equations (108) and jump condition (110) reduce to

$$
\left.\begin{array}{r}
2 \lambda^{\prime} \boldsymbol{t}-(\gamma \boldsymbol{g})^{\prime \prime}+\left(v \boldsymbol{g} \times \boldsymbol{g}^{\prime}\right)^{\prime}+\left(\omega G_{\boldsymbol{d}^{\prime}}\right)^{\prime}=\mathbf{0}, \\
-(v \boldsymbol{t} \times \boldsymbol{g})^{\prime}-v \boldsymbol{t} \times \boldsymbol{g}^{\prime}-2 \chi \boldsymbol{g}+\omega G_{\boldsymbol{g}}-\left(\omega G_{\boldsymbol{g}^{\prime}}\right)^{\prime}=\mathbf{0},
\end{array}\right\}
$$

and

$$
\left.\begin{array}{r}
\llbracket-2 \lambda \boldsymbol{t}+(\gamma \boldsymbol{g})^{\prime}+\left(\nu \boldsymbol{g} \times \boldsymbol{g}^{\prime}\right)^{\prime}+\omega G_{\boldsymbol{d}^{\prime}} \rrbracket=\mathbf{0}, \\
\llbracket \gamma \boldsymbol{g} \rrbracket=\mathbf{0}, \\
\llbracket v \boldsymbol{t} \times \boldsymbol{g}+\omega G_{\boldsymbol{g}^{\prime}} \rrbracket=\mathbf{0} .
\end{array}\right\}
$$

Resolving the components of (116) 2 relative to the basis $\{\boldsymbol{t}, \boldsymbol{g}, \boldsymbol{n}\}$, we obtain three equations which can be viewed as determining $v, \chi$, and $\omega$. In particular, we find from the component along $g$ that $\chi=0$. By $(116)_{1}$, the condition

$$
\begin{aligned}
\gamma(\alpha) \boldsymbol{g}(\alpha)= & \int_{\alpha_{1}}^{\alpha}\left(2 \lambda \boldsymbol{t}+v \boldsymbol{g} \times \boldsymbol{g}^{\prime}+\omega G_{\boldsymbol{d}^{\prime}}\right) \mathrm{d} \alpha+\gamma\left(\alpha_{1}\right) \boldsymbol{g}\left(\alpha_{1}\right) \\
& +\left(\alpha-\alpha_{1}\right)\left(\gamma\left(\alpha_{1}\right) \boldsymbol{g}\left(\alpha_{1}\right)-2 \lambda\left(\alpha_{1}\right) \boldsymbol{t}\left(\alpha_{1}\right)-v\left(\alpha_{1}\right) \boldsymbol{g}\left(\alpha_{1}\right) \times \boldsymbol{g}^{\prime}\left(\alpha_{1}\right)\right. \\
& \left.-\omega\left(\alpha_{1}\right) G_{\boldsymbol{d}^{\prime}}\left(\alpha_{1}\right)\right)
\end{aligned}
$$

must hold for all $\alpha \in\left(\alpha_{1}, \alpha_{2}\right)$ and any choice of the multiplier $\lambda$ compatible with the jump conditions (117) $)_{1}$ and (117) $)_{3}$. Since $\lambda$ is arbitrary on the open interval $\left(\alpha_{1}, \alpha_{2}\right)$, $g$ cannot be determined uniquely from (118). This, however, should not be surprising since the bending energy density in (73) vanishes when $\boldsymbol{d}^{\prime \prime}=\mathbf{0}$, and thus, the distribution of $\boldsymbol{g}$ on a flat portion of $\mathcal{M}$ does not influence the dimensionally reduced bending energy (73). 


\section{Summary and Discussion}

Our analysis is the first study to rigorously demonstrate that the Wunderlich functional (2) is a valid dimensional reduction in the bending energy energy (1) for any closed ribbon $\mathcal{S}$ obtained by an isometric deformation of a flat rectangular strip $\mathcal{S}$ of length $\ell$ and width $2 b$, regardless of the orientability of $\mathcal{S}$ and the number of twists that $\mathcal{S}$ possesses. That finding is, however, predicated on the imposition of the connecting edge conditions (10) and (11), which determine whether or not $\mathcal{S}$ is orientable, and by the corresponding conditions (98) and (101) on the self-linking number of the midline $\mathcal{M}$ of $\mathcal{S}$.

An essential feature of our approach to establishing the validity of the Wunderlich functional (2) involves the referential and spatial directrices. The referential directrix involves not only the midline of the rectangle $\mathcal{D}$ but also segments of its short edges. Hence, the spatial directrix, which is parametrized by $\boldsymbol{d}$, involves not only the midline $\mathcal{M}$ of $\mathcal{S}$ but also the curve on $\mathcal{S}$ that is created by joining the short edges of $\mathcal{D}$ consistent with whichever of the connecting edge conditions (98) and (101) applies. This ensures that both $\mathcal{D}$ and $\mathcal{S}$ are completely covered, and thus, that $\chi$ is surjective. Choosing $\mathcal{M}$ alone as the spatial directrix, as done by Dias and Audoly (2015), does not generally suffice to ensure the satisfaction of that essential requirement.

We find that a closed ribbon $\mathcal{S}$ obtained by minimizing the Wunderlich functional (2) represents the shape determined by a locally injective isometric deformation $\chi$ of a flat rectangular strip $\mathcal{D}$ only if certain additional constraints are met. Those constraints can be expressed in terms of four scalar conditions and one inequality involving the directrix $\boldsymbol{d}$ and generatrix $\boldsymbol{g}$ of $\mathcal{S}$ and their derivatives with respect to arclength along $\mathcal{M}$. This leads us to a constrained variational problem, incorporating four scalar Lagrange multipliers and one Karush-Kuhn-Tucker multiplier, each of which is generally dependent on arclength along $\mathcal{M}$, for the augmented energy functional $E^{*}$ defined in (92). The local injectivity condition is an important ingredient in the literature on isometric immersions with finite bending energy. However, the works in that literature do not include derivations of dimensionally reduced bending energy or the associated boundary value problems for determining the deformation. On the other hand, the local injectivity condition is mostly overlooked in the remainder of the pertinent literature.

Our approach is contingent upon the requirement that the bending energy be finite. Although we allow rulings to intersect on the boundary $\partial \mathcal{S}$ of the ribbon, we do not consider situations where rulings intersect on the interior of $\mathcal{S}$. Granted these provisions, we demonstrate that knowledge of the spatial directrix $\boldsymbol{d}$ and spatial generatrix $g$ suffices to ensure that we can construct a $C^{1}$ surface $\mathcal{S}$ and corresponding deformation $\chi$ that is sufficiently regular to guarantee that the curvature of $\mathcal{S}$ is piecewise continuous.

The Euler-Lagrange equations corresponding to our constrained variational problem consist not only of ordinary differential equations for the directrix $\boldsymbol{d}$ and generatrix $g$ of $\mathcal{S}$ but also of matching conditions that apply at the point on the midline $\mathcal{M}$ of $\mathcal{S}$ where the midline of $\mathcal{D}$ is joined consistent with whichever of the connecting edge conditions (98) and (101) applies and of jump conditions which apply at points on $\mathcal{M}$ where the curvature of $\mathcal{S}$ is discontinuous. Sadowsky's (1930) construction of a 
Möbius band with a single half twist, which yields a surface that is $C^{1}$ but only piecewise $C^{2}$, demonstrates that there do exist configurations of half-twist ribbons that are $C^{1}$ but only piecewise $C^{2}$. This justifies our regularity assumptions on $\boldsymbol{d}$ and $\boldsymbol{g}$. We make no claims about the regularity of the equilibrium configurations, which may even be $C^{2}$. The derivation of our Euler-Lagrange equations uses standard techniques and, in particular, does not require recourse to more sophisticated methods such as the higher-order variational approach of Gay-Balmaz et al. (2012).

To obtain the final form of their Euler-Lagrange equations for the equilibrium shapes of half-twist Möbius bands, Starostin and van der Heijden (2007, 2015) restricted attention to shapes that possess 180-degree rotational symmetry about a particular axis. ${ }^{7}$ They also considered the equilibrium shapes of Möbius bands with multiple half-twists and, to write down the final equations, assumed that the associated shapes posses certain specific symmetries. Although some physical constructions of half-twist Möbius bands appear to possess such symmetry, we are not aware of a proof demonstrating that such symmetry is always achieved in equilibrium. Since our framework does not involve symmetry assumptions, it provides, in particular, a basis for developing numerical methods for investigating the existence and stability of shapes with and without symmetry.

The problem considered here is a special version of a more general one in which displacements and dead loads, constituted by tractions and couple tractions, are applied on the boundary of the ribbon. In such problems, the underlying variational principle must include the potential energy of the dead loads. The task of generalizing the dimensional reduction argument leading to (73) when dead loads are present is the subject of a forthcoming work. Another important direction, which is also in progress, involves extending our results to deal with reference shapes that are not rectangular but which can be smoothly ruled. The most challenging extension of our work arises when a smooth ruling is not possible. This can occur even if the reference shape is rectangular, but its four corners are subjected to mild displacements perpendicular to the plane in which it resides.

Acknowledgements We thank Michael Grunwald for assistance with Fig. 3 and Dmitrii Koldaev for carefully reading and providing feedback on an earlier draft. EF gratefully acknowledges support from the Okinawa Institute of Science and Technology Graduate University with subsidy funding from the Cabinet Office, Government of Japan.

Open Access This article is licensed under a Creative Commons Attribution 4.0 International License, which permits use, sharing, adaptation, distribution and reproduction in any medium or format, as long as you give appropriate credit to the original author(s) and the source, provide a link to the Creative Commons licence, and indicate if changes were made. The images or other third party material in this article are included in the article's Creative Commons licence, unless indicated otherwise in a credit line to the material. If material is not included in the article's Creative Commons licence and your intended use is not permitted by statutory regulation or exceeds the permitted use, you will need to obtain permission directly from the copyright holder. To view a copy of this licence, visit http://creativecommons.org/licenses/by/4.0/.

\footnotetext{
7 This symmetry assumption was also made in the work of Mahadevan and Keller (1993) and Moore and Healey (2019).
} 


\section{References}

Bartels, S., Hornung, P.: Bending paper and the Möbius strip. J. Elast. 119, 113-136 (2015)

Chen, Y.-C., Fosdick, R., Fried, E.: Representation of a smooth isometric deformation of a planar material region into a curved surface. J. Elast. 130, 145-195 (2018a)

Chen, Y.-C., Fosdick, R., Fried, E.: Issues concerning isometric deformations of planar regions to curved surfaces. J. Elast. 132, 1-41 (2018b)

Dias, M.A., Audoly, B.: "Wunderlich, meet Kirchhoff": a general and unified description of elastic ribbons and thin rods. J. Elast. 119, 49-66 (2015)

do Carmo, M.P.: Differential Geometry of Curves and Surfaces. Prentice-Hall, Upper Saddle River (1976)

Gay-Balmaz, F., Holm, D.D., Meier, D.M., Ratiu, T.S., Vialard, F.X.: Invariant higher-order variational problems. Commun. Math. Phys. 309, 413-458 (2012)

Halpern, B., Weaver, C.: Inverting a cylinder through isometric immersions and isometric embeddings. Trans. Am. Math. Soc. 230, 41-70 (1977)

Hartman, P., Nirenberg, L.: On spherical image maps whose Jacobians do not change sign. Am. J. Math. 81(4), 901-920 (1959)

Hartman, P., Wintner, A.: On the asymptotic curves of a surface. Am. J. Math. 73, 149-172 (1951)

Hinz, D.F., Fried, E.: Translation of Michael Sadowsky's paper "An elementary proof for the existence of a developable Möbius band and the attribution of the geometric problem to a variational problem". J. Elast. 119, 3-6 (2015)

Hornung, P.: Fine level set structure of flat isometric immersions. Arch. Ration. Mech. Anal. 199, 943-1014 (2011a)

Hornung, P.: Euler-Lagrange equation and regularity for flat minimizers of the Willmore functional. Commun. Pure Appl. Math. 64, 367-441 (2011b)

Hornung, P.: Approximation of flat $W^{2,2}$ isometric immersions by smooth ones. Arch. Ration. Mech. Anal. 199, 1015-1067 (2011c)

Kirchheim, B.: Rigidity and geometry of microstructures. https:/www.mis.mpg.de/publications/otherseries/ln/lecturenote-1603.html (2003). Accessed 6 May 2020

Mahadevan, L., Keller, J.B.: The shape of a Möbius band. Proc. R. Soc. A Ser. A Math. Phys. Eng. Sci. 440, 149-162 (1993)

Moore, A., Healey, T.: Computation of elastic equilibria of complete Möbius bands and their stability. Math. Mech. Solids 24, 939-967 (2019)

Müller, S., Pakzad, M.R.: Regularity properties of isometric immersions. Math. Z. 251, 313-331 (2005)

Myers, S.B., Steenrod, N.E.: The group of isometries of a Riemannian manifold. Ann. Math. 40, 400-416 (1939)

Pakzad, M.R.: On the Sobolev space of isometric immersions. J. Differ. Geom. 66, 47-69 (2004)

Sadowsky, M.: Ein elementarer Beweis für die Existenz einer abwickelbaren Möbiusschen Bandes und die Zurüchführung des geometrischen Problems auf ein Variationsproblem. Sitzungsberichte der Preussischen Akademie der Wissenschaften, physikalisch-mathematische Klasse 22, 412-415 (1930)

Starostin, E.L., van der Heijden, G.H.M.: The shape of a Möbius strip. Nat. Mater. 6, 563-567 (2007)

Starostin, E.L., van der Heijden, G.H.M.: Equilibrium shapes with stress localisation for inextensible elastic Möbius and other strips. J. Elast. 119, 67-112 (2015)

Todres, R.E.: Translation of W. Wunderlich's, “On a developable Möbius band”. J. Elast. 119, 23-34 (2015)

Wunderlich, W.: Über ein abwickelbares Möbiusband. Monatsh. Math. 66, 276-289 (1962)

Publisher's Note Springer Nature remains neutral with regard to jurisdictional claims in published maps and institutional affiliations. 